\title{
ON PARAMETRIC EVOLUTION INCLUSIONS OF THE SUBDIFFERENTIAL TYPE WITH APPLICATIONS TO OPTIMAL CONTROL PROBLEMS
}

\author{
NIKOLAOS S. PAPAGEORGIOU
}

\begin{abstract}
In this paper we study parametric evolution inclusions of the subdifferential type and their applications to the sensitivity analysis of nonlinear, infinite dimensional optimal control problems. The parameter appears in all the data of the problem, including the subdifferential operator. First we establish several continuity results for the solution multifunction of the subdifferential inclusion. Then we study how these results can be used to examine the sensitivity properties (variational stability) of certain broad classes of nonlinear infinite dimensional optimal control problems. Some examples are worked out in detail, illustrating the applicability of our work. These include obstacle problems (with time varying obstacles), optimal control of distributed parameter systems, and differential variational inequalities.
\end{abstract}

\section{INTRODUCTION}

In this paper we consider parametric nonlinear evolution inclusions of the form

$$
\left\{\begin{array}{c}
-\dot{x}(t) \in \partial \varphi(t, x(t), \lambda)+F(t, x(t), \lambda) \text { a.e. on } T=[0, b] \\
x(0)=x_{0}(\lambda)
\end{array}\right\}
$$

defined in a separable Hilbert space $H$. Here $\partial \varphi(t, \cdot, \lambda)$ is the subdifferential of a proper, lower semicontinuous convex function and $\lambda \in E$ is a parameter taking values in a complete metric space $E$.

Denote by $S(\lambda) \subseteq C(T, H)$ the set of strong solutions of (1) for a given value $\lambda \in E$ of the parameter. Our goal is to investigate the continuity properties of the multifunction (set-valued map) $\lambda \rightarrow S(\lambda)$. Note that the parameter appears in all the data of the evolution, including the subdifferential operator. Furthermore, our subdifferential is in general time-varying and for $s \neq t, \overline{\operatorname{dom} \varphi(s, \cdot, \lambda)}$ need not be equal to $\overline{\operatorname{dom} \varphi(t, \cdot, \lambda)}$. Hence our formulation also incorporates multivalued obstacle problems, with time-varying obstacles as well as differential variational inequalities (see Section 5). The problem of continuous dependence of the solution set on parameters was studied

Received by the editors June 11, 1993.

1991 Mathematics Subject Classification. Primary 34A60, 34G20, 49J24; Secondary 35K55, $35 \mathrm{~L} 85$.

Key words and phrases. Subdifferential, strong solution, $G$-convergence, Mosco convergence, Vietoris topology, Hausdorff metric, optimal control problem, obstacle problem, differential variational inequality. 
earlier for differential inclusions in $\mathbb{R}^{N}$ by Vasilev [29] and Lim [21]. More recently, Kravvaritis-Papageorgiou [19] addressed this issue in the context of timeindependent subdifferential inclusions and, under more restrictive hypotheses than the ones employed here, proved a continuous dependence result (see Theorem 4.1 of [19]). In this paper, under weaker hypotheses on the data, we obtain stronger results, that even guarantee the continuity of the multifunction $\lambda \rightarrow S(\lambda)$ in both the Vietoris and Hausdorff metric hyperspace topologies. Our tools are the $G$-convergence of maximal monotone operators and the Mosco convergence of proper functions. These are the appropriate notions, which allow us to treat distributed parameter systems, with weakly convergent (rapidly changing) coefficients. In Section 4, the continuous dependence results are used to examine the sensitivity properties of a class of infinite dimensional nonlinear optimal control problems. In Section 5, a few examples are worked out in detail.

\section{Preliminaries}

Let $(\Omega, \Sigma)$ be a measurable space, and let $X$ be a separable Banach space. Throughout this work we will be using the following notations:

$$
P_{f(c)}(X)=\{A \subseteq X: \text { nonempty, closed (convex) }\}
$$

and

$$
P_{(w) k(c)}(X)=\{A \subseteq X \text { : nonempty, (weakly-) compact, (convex) }\} .
$$

A multifunction (set-valued function), is said to be measurable if and only if for all $x \in X$, the $\mathbb{R}_{+}$-valued function $\omega \rightarrow d(x, F(\omega))=\inf \{\|x-y\|: y \in$ $F(\omega)\}$ is measurable. Next let $\mu(\cdot)$ be a finite measure defined on $(\Omega, \Sigma)$. By $S_{F}^{p}, 1 \leq p \leq \infty$, we will denote the set of all selectors of $F(\cdot)$ that belong in the Lebesgue-Bochner space $L^{p}(\Omega, X)$; i.e. $S_{F}^{p}=\left\{f \in L^{p}(\Omega, X): f(\omega) \in\right.$ $F(\omega) \mu$-a.e. $\}$. In general this set may be empty. However, using Aumann's selection theorem (see Wagner [30], Theorem 5.10), we can easily check that for a measurable multifunction $F: \Omega \rightarrow P_{f}(X)$, the set $S_{F}^{p}$ is nonempty if and only if $\omega \rightarrow \inf \{\|x\|: x \in F(\omega)\} \in L_{+}^{p}$.

Let $\varphi: X \rightarrow \overline{\mathbb{R}}=\mathbb{R} \cup\{+\infty\}$. We say that $\varphi(\cdot)$ is proper if it is not identically $+\infty$. Assume that $\varphi(\cdot)$ is a proper, convex, and lower semicontinuous (1.s.c.) function (usually this family of $\overline{\mathbb{R}}$-valued functions is denoted by $\left.\Gamma_{0}(X)\right)$. By $\operatorname{dom} \varphi$, we will denote the effective domain of $\varphi(\cdot)$; i.e. $\operatorname{dom} \varphi=\{x \in X: \varphi(x)<+\infty\}$. The subdifferential of $\varphi(\cdot)$ at $x$ is the set $\partial \varphi(x)=\left\{x^{*} \in X^{*}:\left(x^{*}, y-x\right) \leq \varphi(y)-\varphi(x)\right.$ for all $\left.y \in \operatorname{dom} \varphi\right\}$ (in this definition by $(\cdot, \cdot)$ we denote the duality brackets for the pair $\left.\left(X, X^{*}\right)\right)$. It is easy to see that the set $\partial \varphi(x)$ (possibly empty) is convex and $w^{*}$-closed. Furthermore, $\partial \varphi(x) \neq \varnothing$ for all $x \in \operatorname{int} \operatorname{dom} \varphi$ and if $\varphi(\cdot)$ is Gateaux differentiable at $x \in X$, then $\partial \varphi(x)=\left\{\varphi^{\prime}(x)\right\}$. We say that $\varphi \in \Gamma_{0}(X)$ is of the compact type, if for every $\lambda \in \mathbb{R}_{+}$, the level set $\left\{x \in X:\|x\|^{2}+\varphi(x) \leq \lambda\right\}$ is compact.

It is well known that on $P_{f}(X)$ we can define a generalized metric, known in the literature as the Hausdorff metric, by setting for $A, B \in P_{f}(X)$

$$
h(A, B)=\max \left[\sup _{a \in A} d(a, B), \sup _{b \in B} d(b, A)\right]
$$


where $d(a, B)=\inf \{\|a-b\|: b \in B\}$ and $d(b, A)=\inf \{\|b-a\|: a \in A\}$. The metric space $\left(P_{f}(X), h\right)$ is complete and $\left(P_{k}(X), h\right)$ is a closed, separable subspace of it. If $V$ is a Hausdorff topological space, a multifunction $F: V \rightarrow$ $P_{f}(X)$ is said to be Hausdorff continuous ( $h$-continuous), if it is continuous from $V$ into the metric space $\left(P_{f}(X), h\right)$.

Let $Y, Z$ be Hausdorff topological spaces and $G: Y \rightarrow 2^{Z} \backslash\{\varnothing\}$. We say that $G(\cdot)$ is upper semicontinuous (u.s.c.) (resp. lower semicontinuous (1.s.c.)) if for all $U \subseteq Z$ open the set $G^{+}(U)=\{y \in Y: G(y) \subseteq U\}$ (resp. $G^{-}(U)=$ $\{y \in Y: G(y) \cap U \neq \varnothing\})$ is open in $Y$. A multifunction $G(\cdot)$ which is both u.s.c. and l.s.c. is said to be continuous or Vietoris continuous, to emphasize that on $2^{Z} \backslash\{\varnothing\}$ we are considering the Vietoris hyperspace topology.

Finally let us introduce the two convergence concepts for functionals and operators mentioned in the introduction. Let $\left\{\varphi_{n}, \varphi\right\}_{n \geq 1} \subseteq \Gamma_{0}(X)$. We define

$$
\text { epi } \varphi_{n}=\left\{(x, \lambda) \in X \times \mathbb{R}: \varphi_{n}(x) \leq \lambda\right\}
$$

(the epigraph of $\varphi_{n}$ ). We say that the $\varphi_{n}$ 's converge to $\varphi$ in the Mosco sense (denoted by $\varphi_{n} \stackrel{M}{\rightarrow} \varphi$ ) if and only if

$$
w \text { - }
$$

where $s$-lim epi $\varphi_{n}=\left\{(x, \lambda) \in X \times \mathbb{R}:(x, \lambda)=\lim \left(x_{n}, \lambda_{n}\right),\left(x_{n}, \lambda_{n}\right) \in \operatorname{epi} \varphi_{n}\right.$, $n \geq 1\}$ and $w-\varlimsup \lim \varphi_{n}=\left\{(x, \lambda) \in X \times \mathbb{R}:(x, \lambda)=w-\lim \left(x_{n_{k}}, \lambda_{n_{k}}\right),\left(x_{n_{k}}, \lambda_{n_{k}}\right) \in\right.$ epi $\left.\varphi_{n_{k}}, n_{1}<n_{2}<\cdots<n_{k}<\cdots\right\}$ (where $w$ denotes the weak topology on $X \times \mathbb{R})$. It is clear from these definitions that we always have $s$ - $\underline{\lim } \operatorname{epi} \varphi_{n} \subseteq$ $w$-Пim epi $\varphi_{n}$. So the inclusions in the above definition of Mosco convergence, are in fact equalities. It is also easy to see that $\varphi_{n} \stackrel{M}{\rightarrow} \varphi$ as $n \rightarrow \infty$ if and only if the following two conditions hold for every $x \in X$ :

(i) for every $x_{n} \stackrel{w}{\rightarrow} x, \varphi(x) \leq \underline{\lim } \varphi_{n}\left(x_{n}\right)$, and

(ii) there exists $x_{n} \rightarrow x$ such that $\varphi(x)=\lim \varphi_{n}\left(x_{n}\right)$.

This concept of convergence of functionals was first introduced by Mosco [23] (Definition 1.4) as the appropriate notion of variational convergence, to study the convergence properties of the solutions of a sequence of variational inequalities. Note that $\varphi_{n} \stackrel{M}{\rightarrow} \varphi$ if and only if $\Gamma_{s e q}(X) \varphi_{n}=\varphi$ and $\Gamma_{s e q}\left(X_{w}\right) \varphi_{n}=$ $\varphi$, where $\Gamma_{\text {seq }}$ denotes the notion of sequential $\Gamma$-convergence and $X_{w}$ denotes the Banach space equipped with the weak topology. For further details on the concepts of $M$ - and $\Gamma$-convergence, we refer to the well-written monographs of Attouch [2] and Dal Maso [9].

Let $Z$ be a reflexive Banach space and $\left\{A_{n}\right\}_{n \geq 1}$ a sequence of maximal monotone operators from $Z$ into $2^{Z^{*}}$. We define $\operatorname{Gr} A_{n}=\{(x, y) \in Z \times$ $\left.Z^{*}: y \in A_{n}(x)\right\}$. We say that the $A_{n}$ 's $G$-converge to $A$ (denoted by $A_{n} \stackrel{G}{\rightarrow} A$ ) if and only if

$$
\varlimsup \lim \operatorname{Gr} A_{n} \subseteq \mathrm{Gr} A \subseteq \underline{\lim } \mathrm{Gr} A
$$

where

$\varliminf \operatorname{Gr} A_{n}=\left\{(x, y) \in Z \times Z^{*}:(x, y)=\lim \left(x_{n}, y_{n}\right),\left(x_{n}, y_{n}\right) \in \mathrm{Gr} A_{n}, n \geq 1\right\}$ and

$\varlimsup \lim \operatorname{Gr} A_{n}=\left\{(x, y) \in Z \times Z^{*}:(x, y)=\lim \left(x_{n_{k}}, y_{n_{k}}\right),\left(x_{n_{k}}, y_{n_{k}}\right) \in \operatorname{Gr} A_{n_{k}}\right.$, $\left.n_{1}<n_{2}<\cdots<n_{k}<\cdots\right\}$. 
As before, we always have that $\underline{\lim } \operatorname{Gr} A_{n} \subseteq \varlimsup_{\lim } \operatorname{Gr} A_{n}$ and so the inclusions in the definition of $G$-convergence are in fact equalities. For further details we refer to Attouch [2]. Recall that the subdifferential $\partial \varphi(x)$ of a function $\varphi \in \Gamma_{0}(Z)$ is a maximal monotone operator (see Brezis [6], p. 25).

Now let $T=[0, b]$ and $H$ be a separable Hilbert space. By a "strong solution" of (1), we mean a function $x(\cdot) \in C(T, H)$ such that $x(\cdot)$ is absolutely continuous on any closed subinterval of $(0, b), x(t) \in \operatorname{dom} \varphi(t, \cdot, \lambda)$ a.e., and $-\dot{x}(t) \in \partial \varphi(t, x(t), \lambda)+f(t)$ a.e., $x(0)=x_{0}$ with $f \in S_{F(\cdot, x(\cdot), \lambda)}^{2}$. Recall that an absolutely continuous function $x:(0, b) \rightarrow H$ is strongly differentiable almost everywhere (see Brezis [6], Corollary A.2, p. 145), and so in the above inclusion $\dot{x}(\cdot)$ is the strong derivative of $x(\cdot)$. Let $E$ be a complete metric space (the parameter space).

The following hypothesis concerning $\varphi(t, x, \lambda)$ will be valid throughout this paper:

$H(\varphi): \varphi: T \times H \times E \rightarrow \overline{\mathbb{R}}=\mathbb{R} \cup\{+\infty\}$ is an integrand such that

(1) for every $t \in T \backslash N, \mu(N)=0$ (here $\mu(\cdot)$ denotes the Lebesgue measure on $T$ ) and $0 \notin N$, and for every $\lambda \in E, \varphi(t, \cdot, \lambda)$ is a proper, convex, l.s.c. function (i.e. $\varphi(t, \cdot, \lambda) \in \Gamma_{0}(H)$ ),

(2) for every positive integer $r$, there exists a constant $K_{r}>0$, an absolutely continuous function $g_{r}: T \rightarrow \mathbb{R}$ with $\dot{g}_{r} \in L^{\beta}(T, \mathbb{R})$, and a function of bounded variation $h_{r}: T \rightarrow \mathbb{R}$ such that if $t \in T \backslash N, \lambda \in$ $E, x \in \operatorname{dom} \varphi(t, \cdot, \lambda)$ with $\|x\| \leq r$ and $s \in[t, b] \backslash N$, then we can find $\hat{x} \in \operatorname{dom} \varphi(s, \cdot, \lambda)$ satisfying

$$
\begin{gathered}
\|\hat{x}-x\| \leq\left|g_{r}(s)-g_{r}(t)\right|\left(\varphi(t, x, \lambda)+K_{r}\right)^{\alpha}, \\
\varphi(s, \hat{x}, \lambda) \leq \varphi(t, x, \lambda)+\left|h_{r}(s)-h_{r}(t)\right|\left(\varphi(t, x, \lambda)+K_{r}\right)
\end{gathered}
$$

with $\alpha \in[0,1]$ and $\beta=2$ if $\alpha \in\left[0, \frac{1}{2}\right]$, or $\beta=\frac{1}{1-\alpha}$ if $\alpha \in\left[\frac{1}{2}, 1\right]$,

(3) if $\lambda_{n} \rightarrow \lambda$ in $E$, then $\varphi\left(t, \cdot, \lambda_{n}\right) \stackrel{M}{\rightarrow} \varphi(t, \cdot, \lambda)$ for all $t \in T \backslash N$.

Remark. Hypotheses $H(\varphi)(1)$ and (2) are essentially due to Kenmochi [15] and Yamada [31]. Here we use a slightly generalized version introduced by Yotsutani [32]. In particular, in Kenmochi [15], $N=\varnothing$ and $g_{r}, h_{r}$ are both Lipschitz, while in Yamada [31], $N=\varnothing$ and $h_{r}$ is absolutely continuous. We should remark that Yamada [31] and Kenmochi [16] were the first to provide some important applications to partial differential equations of evolution equations generated by time dependent subdifferentials. Hypothesis $H(\varphi)(3)$ refers to the dependence on the parameter $\lambda \in E$ of the functional $\varphi(t, x, \lambda)$ and is equivalent to the $G$-convergence of the subdifferential operator plus a normalization condition (see Attouch [2], p. 373).

\section{Continuous DEPENDENCE RESULTS}

In this section we prove several continuous dependence results for problem (1) (see Section 1). Recall that by $S(\lambda) \subseteq C(T, H)$ we denote the set of strong solutions of (1) corresponding to the parameter $\lambda \in E$. Our first result shows that the multifunction $\lambda \rightarrow S(\lambda)$ has a closed graph (i.e. if $\lambda_{n} \rightarrow \lambda$ in $E$, then $\varlimsup \lim S\left(\lambda_{n}\right)=\left\{x \in C(T, H): x=\lim x_{n_{k}}, x_{n_{k}} \in S\left(\lambda_{n_{k}}\right), n_{1}<n_{2}<\cdots<n_{k}<\right.$ $\cdots\} \subseteq S(\lambda))$. For this, we will need the following hypothesis on the orientor field $F(t, x, \lambda)$ : 
$H(F)_{1}: F: T \times H \times E \rightarrow P_{w k c}(H)$ is a multifunction such that

(1) $\operatorname{Gr} F(\cdot, \cdot, \lambda)=\{(t, x, v) \in T \times H \times H: v \in F(t, x, \lambda)\} \in B(T) \times$ $B(H) \times B(H)$, where $B(T)$ (resp. $B(H)$ ) is the Borel $\sigma$-field of $T$ (resp. of $H$ ) (graph measurability of $F(\cdot, \cdot, \lambda)$ ),

(2) $\operatorname{Gr} F(t, \cdot, \cdot)=\{(x, \lambda, v) \in H \times E \times H: v \in F(t, x, \lambda)\}$ is sequentially closed in $H \times E \times H_{w}$, where $H_{w}$ denotes the Hilbert space $H$ furnished with the weak topology,

(3) $|F(t, x, \lambda)|=\sup \{\|v\|: v \in F(t, x, \lambda)\} \leq a_{B}(t)+c_{B}(t)\|x\|$ a.e. for all $\lambda \in B \subseteq E$ compact and with $a_{B}, c_{B} \in L_{+}^{2}$.

Remark. Hypothesis $H(F)_{1}(1)$ is weaker than assuming that $(t, x) \rightarrow F(t, x, \lambda)$ is measurable (see Wagner [30], Theorem 4.2), while hypothesis $H(F)_{1}(2)$ is weaker than assuming that $(x, \lambda) \rightarrow F(t, x, \lambda)$ is u.s.c. from $H \times E$ into $H_{w}$ (see DeBlasi-Myjak [11], Remark 1.4).

To guarantee that $S(\lambda)$ is nonempty for every $\lambda \in E$, we will need the following hypothesis:

$H_{c}$ : One of the following two conditions holds:

(1) for every $t \in T \backslash N$ and every $\lambda \in E, \varphi(t, \cdot, \lambda)$ is of compact type,

(2) for every $(t, \lambda) \in T \times E$ and every $K \subseteq H$ bounded, $\overline{F(t, K, \lambda)}$ is compact in $H$.

Remark. These are compactness type conditions, the first imposed on the functional $\varphi(t, x, \lambda)$ and the second on the orientor field $F(t, x, \lambda)$. Both imply that for every $\lambda \in E, S(\lambda)$ is a nonempty and compact subset of $C(T, H)$ (see Papageorgiou [26], [27]).

Finally, our hypothesis on the initial condition is the following:

$H_{0}: \lambda \rightarrow x_{0}(\lambda)$ is continuous from $E$ into $H$, for every $\lambda \in E$ we have $x_{0}(\lambda) \in$ $\operatorname{dom} \varphi(0, \cdot, \lambda)$, and for every $B \subseteq E$ compact we have $\sup _{\lambda \in B} \varphi\left(0, x_{0}(\lambda), \lambda\right)<$ $\infty$.

Proposition 3.1. If hypotheses $H(\varphi), H(F)_{1}, H_{c}$, and $H_{0}$ hold, then the multifunction $\lambda \rightarrow S(\lambda)$ has a closed graph in $E \times C(T, H)$ (i.e. if $\lambda_{n} \rightarrow \lambda$ in $E$, then $\left.\overline{\lim } S\left(\lambda_{n}\right) \subseteq S(\lambda)\right)$.

Proof. Let $x_{n} \in S\left(\lambda_{n}\right), n \geq 1$, and assume that $\lambda_{n} \rightarrow \lambda$ in $E$ and $x_{n} \rightarrow x$ in $C(T, H)$. By definition we have

$$
\left\{\begin{array}{c}
-\dot{x}_{n}(t) \in \partial \varphi\left(t, x_{n}(t), \lambda_{n}\right)+f_{n}(t) \text { a.e. } \\
x_{n}(0)=x_{0}\left(\lambda_{n}\right)
\end{array}\right\}
$$

with $f_{n} \in L^{2}(T, H), f_{n}(t) \in F\left(t, x_{n}(t), \lambda_{n}\right)$ a.e. Let $\Phi: L^{2}(T, H) \times E \rightarrow \overline{\mathbb{R}}=$ $\mathbb{R} \cup\{+\infty\}$ be the integral functional defined by

$$
\Phi(x, \lambda)= \begin{cases}\int_{0}^{b} \varphi(t, x(t), \lambda) d t & \text { if } \varphi(\cdot, x(\cdot), \lambda) \in L^{1}(T, \mathbb{R}), \\ +\infty & \text { otherwise. }\end{cases}
$$

From Lemma 3.4 of Yotsutani [32], we know that $t \rightarrow \varphi(t, x(t), \lambda)$ is measurable. So $\Phi(x, \lambda)$ is well defined. Furthermore from Lemma 4.4 of Yotsutani [32], we know that if $v \in L^{2}(T, H), \varphi(\cdot, v(\cdot), \lambda) \in L^{1}(T, \mathbb{R})$, and 
$w \in L^{2}(T, H)$, then $w \in \partial \Phi(v, \lambda)$ if and only if $w(t) \in \partial \varphi(t, v(t), \lambda)$ a.e. on $T$. So we have

$$
\left[x_{n},-\dot{x}_{n}-f_{n}\right] \in \operatorname{Gr} \partial \Phi\left(\cdot, \lambda_{n}\right) .
$$

Next we will show that our hypothesis $H(\varphi)(3)$ implies that $\partial \Phi\left(\cdot, \lambda_{n}\right) \stackrel{G}{\rightarrow}$ $\partial \Phi(\cdot, \lambda)$ in $L^{2}(T, H) \times L^{2}(T, H)$. Indeed note that for every $v \in L^{2}(T, H)$ and almost all $t \in T$, we have

$$
\left[\left(I+\partial \Phi\left(\cdot, \lambda_{n}\right)\right)^{-1} v\right](t)=\left(I+\partial \varphi\left(t, \cdot, \lambda_{n}\right)\right)^{-1}(v(t)) .
$$

Furthermore from Lemma 3.3 of Yotsutani [32], we know that for all $n \geq 1$ and all $t \in T \backslash N$

$$
\left\|\left(I+\partial \varphi\left(t, \cdot, \lambda_{n}\right)\right)^{-1}(v(t))\right\| \leq 2\|v(t)\|+c
$$

with $c>0$. Thus applying the dominated convergence theorem, we have

$$
\left(I+\partial \Phi\left(\cdot, \lambda_{n}\right)\right)^{-1} v \rightarrow(I+\partial \Phi(\cdot, \lambda))^{-1} v \quad \text { in } L^{2}(T, H)
$$

and this by Proposition 3.60, p. 361 of Attouch [2], implies that

$$
\partial \Phi\left(\cdot, \lambda_{n}\right) \stackrel{G}{\rightarrow} \partial \Phi(\cdot, \lambda) \quad \text { as } n \rightarrow \infty
$$

in $L^{2}(T, H) \times L^{2}(T, H)$.

Note that $f_{n}(t) \in F\left(t, x_{n}(t), \lambda_{n}\right)$ a.e. and because of hypothesis $H(F)_{1}(3)$ and with $B=\left\{\lambda_{n}, \lambda\right\}_{n \geq 1} \subseteq E$ we have $\left\|f_{n}(t)\right\| \leq a_{B}(t)+c_{B}(t) M_{1}=\psi_{n}(t)$ a.e. with $M_{1}>0$ such that $\left\|x_{n}\right\|_{C(T, H)} \leq M_{1}$ for all $n \geq 1$. So by passing to a subsequence if necessary, we may assume that $f_{n} \stackrel{w}{\rightarrow} f$ in $L^{2}(T, H)$. Using Theorem 3.1 of Papageorgiou [24], we get

$$
f(t) \in \overline{\operatorname{conv}} w-\overline{\lim } F\left(t, x_{n}(t), \lambda_{n}\right) \text { a.e. }
$$

Because of hypothesis $H(F)_{1}(2)$, we know that

$$
\overline{\operatorname{conv}} w-\overline{\lim } F\left(t, x_{n}(t), \lambda_{n}\right) \subseteq F(t, x(t), \lambda) \text { a.e. }
$$

Also from Yotsutani [32], inequality (7.2), p. 645, and because of hypotheses $H(F)_{1}(3)$ and $H_{0}$, we have

$$
\sup _{n \geq 1}\left\|\dot{x}_{n}\right\|_{L^{2}(T, H)}=M_{2}<\infty .
$$

So we may assume that $\dot{x}_{n} \stackrel{w}{\rightarrow} y$ in $L^{2}(T, H)$ and clearly $y=\dot{x}$. Hence we have

$$
\left[x_{n},-\dot{x}_{n}-f_{n}\right] \rightarrow[x,-\dot{x}-f] \text { as } n \rightarrow \infty
$$

in $L^{2}(T, H) \times L^{2}(T, H)_{w}$ and for every $n \geq 1,\left[x_{n},-\dot{x}_{n}-f_{n}\right] \in \operatorname{Gr} \partial \Phi\left(\cdot, \lambda_{n}\right)$. Since $\partial \Phi\left(\cdot, \lambda_{n}\right) \stackrel{G}{\rightarrow} \partial \Phi(\cdot, \lambda)$, using Proposition 3.59, p. 361 of Attouch [2], we deduce that

$$
\begin{aligned}
& {[x,-\dot{x}-f] \in \operatorname{Gr} \partial \Phi(\cdot, \lambda)} \\
& \quad \Rightarrow-\dot{x}(t) \in \partial \varphi(t, x(t), \lambda)+f(t) \text { a.e., } x(0)=x_{0}(\lambda)
\end{aligned}
$$

with $f(\cdot) \in L^{2}(T, H), f(t) \in F(t, x(t), \lambda)$ a.e. Therefore $x \in S(\lambda)$ and so we conclude that $\lambda \rightarrow S(\lambda)$ has a closed graph in $E \times C(T, H)$.

Next we will establish the lower semicontinuity of the multifunction $\lambda \rightarrow$ $S(\lambda)$. So our result tells us that if $\lambda_{n} \rightarrow \lambda$ in $E$ and $x \in S(\lambda)$, then we can 
always find $x_{n} \in S\left(\lambda_{n}\right), n \geq 1$, such that $x_{n} \rightarrow x$ in $C(T, H)$. For this we will need the following hypothesis on the orientor field $F(t, x, \lambda)$.

$H(F)_{2}: F: T \times H \times E \rightarrow P_{w k c}(H)$ is a multifunction such that

(1) $t \rightarrow F(t, x, \lambda)$ is measurable,

(2) $h(F(t, x, \lambda), F(t, y, \lambda)) \leq k_{B}(t)\|x-y\|$ a.e. for all $\lambda \in B \subseteq E$ compact and with $k_{B} \in L_{+}^{1}$,

(3) $|F(t, x, \lambda)|=\sup \{\|v\|: v \in F(t, x, \lambda)\} \leq a_{B}(t)+c_{B}(t)\|x\|$ a.e. for all $\lambda \in B \subseteq E$ compact with $a_{B}, c_{B} \in L_{+}^{2}$,

(4) $\lambda \rightarrow F(t, x, \lambda)$ is 1.s.c.

Remark. Hypotheses $H(F)_{2}(1)$ and (2) and Theorem 3.3 of Papageorgiou [25] imply that $(t, x) \rightarrow F(t, x, \lambda)$ is measurable.

Proposition 3.2. If hypotheses $H(\varphi), H(F)_{2}, H_{c}$, and $H_{0}$ hold, then $\lambda \rightarrow S(\lambda)$ is a l.s.c. multifunction from $E$ into $P_{k}(C(T, H))$.

Proof. Since both $E$ and $C(T, H)$ are metric spaces, we need to show that if $\lambda_{n} \rightarrow \lambda$ in $E$, then $S(\lambda) \subseteq \lim S\left(\lambda_{n}\right)=\left\{x \in C(T, H): x=\lim x_{n}, x_{n} \in\right.$ $\left.S\left(\lambda_{n}\right), n \geq 1\right\}$ (see DeBlasi-Myjak [11]). To this end let $x(\cdot) \in S(\lambda)$. Then by definition, we have

$$
-\dot{x}(t) \in \partial \varphi(t, x(t), \lambda)+f(t) \text { a.e., } \quad x(0)=x_{0}(\lambda)
$$

with $f \in L^{2}(T, H), f(t) \in F(t, x(t), \lambda)$ a.e.

Set

$$
v\left(t, \lambda_{n}\right)=\operatorname{proj}\left(f(t) ; F\left(t, x(t), \lambda_{n}\right)\right)
$$

and

$$
u\left(t, z, \lambda_{n}\right)=\operatorname{proj}\left(v(t, \lambda) ; F\left(t, z, \lambda_{n}\right)\right)
$$

(here by $\operatorname{proj}(\cdot ; F(t, z, \lambda))$ we denote the metric projection on the set $F(t, z, \lambda),(t, z, \lambda) \in T \times H \times E$; it is well known that this is a single-valued, nonexpansive map). Recall that $(t, x) \rightarrow F\left(t, x, \lambda_{n}\right)$ is measurable (see the remark following hypothesis $\left.H(F)_{2}\right)$. So $t \rightarrow F\left(t, x(t), \lambda_{n}\right)$ is measurable. Then Gr $v\left(\cdot, \lambda_{n}\right)=\left\{(t, h) \in \mathrm{Gr} F\left(\cdot, x(\cdot), \lambda_{n}\right):\|h-f(t)\|=d\left(f(t), F\left(t, x(t), \lambda_{n}\right)\right)\right\}$ $\in \mathscr{L}(T) \times B(H)$, with $\mathscr{L}(T)$ the Lebesgue $\sigma$-field of $T$. Thus $t \rightarrow v\left(t, \lambda_{n}\right)$ is measurable and similarly we get that $t \rightarrow u\left(t, z, \lambda_{n}\right)$ is measurable. In addition from hypothesis $H(F)_{2}(2)$ and Theorem 3.33, p. 322 of Attouch [2], we have that $z \rightarrow u\left(t, z, \lambda_{n}\right)$ is continuous. Then consider the following Cauchy problem:

$$
\left\{\begin{array}{c}
-\dot{z}(t) \in \partial \varphi\left(t, z(t), \lambda_{n}\right)+u\left(t, z(t), \lambda_{n}\right) \text { a.e. } \\
z(0)=x_{0}\left(\lambda_{n}\right)
\end{array}\right\} .
$$

From the existence results of [26] (if $H_{c}(1)$ is valid) and [27] (if $H_{c}(2)$ is valid), we have that the above Cauchy problem has at least one strong solution $x_{n}(\cdot) \in C(T, H)$. Clearly $x_{n}(\cdot) \in S\left(\lambda_{n}\right), n \geq 1$. Let $y_{n}(\cdot)$ be the unique strong solution of $-\dot{y}_{n}(t) \in \partial \varphi\left(t, y_{n}(t), \lambda_{n}\right)+f(t)$ a.e., $y_{n}(0)=x_{0}\left(\lambda_{n}\right)$. Exploiting the monotonicity of the subdifferential operator, we have:

$$
\begin{array}{r}
\left(-\dot{x}_{n}(t)+\dot{y}_{n}(t), y_{n}(t)-\dot{x}_{n}(t)\right) \leq\left(u\left(t, x_{n}(t), \lambda_{n}\right)-f(t), y_{n}(t)-x_{n}(t)\right) \text { a.e. } \\
\quad \Rightarrow \frac{1}{2} \frac{d}{d t}\left\|x_{n}(t)-y_{n}(t)\right\|^{2} \leq\left\|u\left(t, x_{n}(t), \lambda_{n}\right)-f(t)\right\| \cdot\left\|y_{n}(t)-x_{n}(t)\right\| \text { a.e. } \\
\left.\Rightarrow \frac{1}{2}\left\|x_{n}(t)-y_{n}(t)\right\|^{2} \leq \int_{0}^{t}\left\|u\left(s, x_{n}(s), \lambda_{n}\right)-f(s)\right\| \cdot \| y_{(} s\right)-x_{n}(s) \| d s .
\end{array}
$$


Applying Lemma A.5, p. 157 of Brezis [6], we get

$$
\left\|y_{n}(t)-x_{n}(t)\right\| \leq \int_{0}^{t}\left\|u\left(s, x_{n}(s), \lambda_{n}\right)-f(s)\right\| d s .
$$

Remark that

$$
\begin{aligned}
\| u(s, & \left.x_{n}(s), \lambda_{n}\right)-f(s) \| \\
& \leq\left\|u\left(s, x_{n}(s), \lambda_{n}\right)-v\left(s, \lambda_{n}\right)\right\|+\left\|v\left(s, \lambda_{n}\right)-f(s)\right\| \\
& \leq h\left(F\left(s, x(s), \lambda_{n}\right), F\left(s, x_{n}(s), \lambda_{n}\right)\right)+d\left(f(s), F\left(s, x(s), \lambda_{n}\right)\right) \\
& \leq k_{B}(s)\left\|x(s)-x_{n}(s)\right\|+d\left(f(s), F\left(s, x(s), \lambda_{n}\right)\right) \text { a.e. }
\end{aligned}
$$

with $B=\left\{\lambda_{n}, \lambda\right\}_{n \geq 1} \subseteq E$. So we have

$$
\left\|y_{n}(t)-x_{n}(t)\right\| \leq \int_{0}^{b} d\left(f(s), F\left(s, x(s), \lambda_{n}\right)\right) d s+\int_{0}^{t} k_{B}(s)\left\|x_{n}(s)-x(s)\right\| d s .
$$

Because of hypothesis $H(F)_{2}(4), \lambda \rightarrow d(f(s), F(s, x(s), \lambda))$ is an upper semicontinuous function and so from Fatou's lemma and recalling that $f(s) \in$ $F(s, x(s), \lambda)$ a.e., we have

$$
\lim \int_{0}^{b} d\left(f(s), F\left(s, x(s), \lambda_{n}\right)\right) d s=0 .
$$

Also from hypotheses $H(\varphi)(3)$ and $H_{0}$, we have that $y_{n} \rightarrow x$ in $C(T, H)$ (cf. Attouch [2]). Therefore given $\varepsilon>0$, we can find $n_{0}(\varepsilon) \geq 1$ such that for $n \geq n_{0}(\varepsilon)$ we have

$$
\left\|y_{n}(t)-x_{n}(t)\right\| \leq \varepsilon+\int_{0}^{t} k_{B}(s)\left\|y_{n}(s)-x_{n}(s)\right\| d s, \quad t \in T .
$$

Invoking Gronwall's inequality, we get that

$$
\begin{aligned}
& \left\|y_{n}(t)-x_{n}(t)\right\| \leq \varepsilon \exp \left\|k_{B}\right\|_{1} \text { for all } n \geq n_{0}(\varepsilon) \text { and all } t \in T, \\
& \Rightarrow x_{n} \rightarrow x \text { in } C(T, H) .
\end{aligned}
$$

Since $x_{n}(\cdot) \in S\left(\lambda_{n}\right)$, we conclude that $\lambda \rightarrow S(\lambda)$ is 1.s.c.

Combining Propositions 3.1 and 3.2, we can state our first continuity result. We will say that $\lambda \rightarrow S(\lambda)$ is " $K$-continuous" from $E$ into $P_{k}(C(T, H))$, if for every sequence $\lambda_{n} \rightarrow \lambda$ in $E$, we have that $S\left(\lambda_{n}\right) \stackrel{K}{\rightarrow} S(\lambda)$ in $C(T, H)$, where $\stackrel{K}{\rightarrow}$ denotes the classical Kuratowski convergence of sets; i.e. $S(\lambda)=$ $\underline{\lim } S\left(\lambda_{n}\right)=\left\{x \in C(T, H): x=\lim x_{n}, x_{n} \in S\left(\lambda_{n}\right), n \geq 1\right\}=\varlimsup \lim S\left(\lambda_{n}\right)=\{x \in$ $\left.\bar{C}(T, H): x=\lim x_{n_{k}}, x_{n_{k}} \in S\left(\lambda_{n_{k}}\right), n_{1}<n_{2}<\cdots<n_{k}<\cdots\right\}$ (see Attouch [2], Corollary 1.35, p. 95, and Dal Maso [9], Remark 8.2, p. 88). To establish the $K$-continuity of $\lambda \rightarrow S(\lambda)$ we will need the following hypothesis on the orientor field.

$H(F)_{3}: F: T \times H \times E \rightarrow P_{w k c}(H)$ is a multifunction such that

(1) $t \rightarrow F(t, x, \lambda)$ is measurable,

(2) $h(F(t, x, \lambda), F(t, y, \lambda)) \leq k_{B}(t)\|x-y\|$ a.e. for all $\lambda \in B \subseteq E$ compact and with $k_{B} \in L_{+}^{1}$,

(3) $|F(t, x, \lambda)|=\sup \{\|v\|: v \in F(t, x, \lambda)\} \leq a_{B}(t)+c_{B}(t)\|x\|$ a.e. for all $\lambda \in B \subseteq E$ compact and with $a_{B}, c_{B} \in L_{+}^{2}$,

(4) $\lambda \rightarrow F(t, x, \lambda)$ is $h$-continuous. 
Directly from Propositions 3.1 and 3.2, we get the following theorem.

Theorem 3.3. If hypotheses $H(\varphi), H(F)_{3}, H_{c}$, and $H_{0}$ hold, then $\lambda \rightarrow S(\lambda)$ is $K$-continuous from $E$ into $P_{k}(C(T, H))$.

Next we turn our attention to the Vietoris and Hausdorff continuities of the multifunction $\lambda \rightarrow S(\lambda)$. Both are stronger continuity concepts than $K$ continuity and so we have to strengthen our hypotheses to achieve them. More precisely, we will need the following stronger version of hypothesis $H_{c}$ :

$H_{c}^{1}$ : One of the following two conditions holds:

(1) for every $t \in T \backslash N$, for every $B \subseteq E$ compact, and for every $\theta \in \mathbb{R}_{+}$we have that $\bigcup_{\lambda \in B}\left\{z \in H:\|z\|^{2}+\varphi(t, z, \lambda) \leq \theta\right\}$ is compact in $H$ (i.e., $\{\varphi(t, \cdot, \lambda)\}_{\lambda \in B}$ is uniformly of compact type),

(2) for every $t \in T \backslash N$, every $B \subseteq E$ compact, and every $K \subseteq H$ bounded, $\bigcup_{\lambda \in B} F(t, K, \lambda)$ is compact in $H$ (i.e., $\{F(t, \cdot, \lambda)\}_{\lambda \in B}$ is a collectively compact family of multifunctions).

We will need the following simple lemma.

Lemma 3.4. If $X$ is a Banach space, $\left\{x_{n}^{*}, x^{*}\right\}_{n \geq 1} \subseteq X^{*}$, and $x_{n}^{*} \stackrel{w^{*}}{\rightarrow} x^{*}$, then $\left(x_{n}^{*}, u\right) \rightarrow\left(x^{*}, u\right)$ uniformly for all $u \in K \subseteq X$ compact.

Remark. In the above lemma, $\stackrel{w^{*}}{\rightarrow}$ denotes convergence in the $w^{*}$-topology and $(\cdot, \cdot)$ denotes the duality brackets for the pair $\left(X, X^{*}\right)$.

Proof. Let $M>0$ be such that $\left\|x_{n}^{*}\right\|,\left\|x^{*}\right\| \leq M$. Since $K$ is compact, it is totally bounded. So given $\varepsilon>0$, we can find $\left\{u_{1}, \ldots, u_{N}\right\} \subseteq K$ such that $K \subseteq \bigcup_{k=1}^{N} \stackrel{\circ}{B}\left(u_{k}, \frac{\varepsilon}{3 M}\right)$, where $\stackrel{\circ}{B}\left(u_{k}, \frac{\varepsilon}{3 M}\right)=\left\{v \in X:\left\|v-u_{k}\right\|<\frac{\varepsilon}{3 M}\right\}$. Note that for every $k \in\{1,2, \ldots, N\},\left(x_{n}^{*}, u_{k}\right) \rightarrow\left(x^{*}, u_{k}\right)$ as $n \rightarrow \infty$. So we can find $n_{0 k} \geq 1$ such that for all $n \geq n_{0 k}$ we have $\left|\left(x_{n}^{*}, u_{k}\right)-\left(x^{*}, u_{k}\right)\right|<\frac{\varepsilon}{3}$. Let $\hat{n}_{0}=\max \left\{n_{0 k}\right\}_{k=1}^{N}$. Let $u \in K$ and let $k \in\{1, \ldots, N\}$ be such that $u \in \stackrel{\circ}{B}\left(u_{k}, \frac{\varepsilon}{3 M}\right)$. We have for $n \geq \hat{n}_{0}$

$$
\begin{aligned}
\left|\left(x_{n}^{*}, u\right)-\left(x^{*}, u\right)\right| \leq & \left|\left(x_{n}^{*}, u\right)-\left(x_{n}^{*}, u_{k}\right)\right| \\
& +\left|\left(x_{n}^{*}, u_{k}\right)-\left(x^{*}, u_{k}\right)\right|+\left|\left(x^{*}, u_{k}\right)-\left(x^{*}, u\right)\right| \\
< & M \frac{\varepsilon}{3 M}+\frac{\varepsilon}{3}+M \frac{\varepsilon}{3 M}=\varepsilon
\end{aligned}
$$

$\Rightarrow\left(x_{n}^{*}, u\right) \rightarrow\left(x^{*}, u\right)$ uniformly for $u \in K$.

Now we can prove the following continuous dependence result:

Theorem 3.5. If hypotheses $H(\varphi), H(F)_{3}, H_{c}^{1}$, and $H_{0}$ hold, then the multifunction $\lambda \rightarrow S(\lambda)$ is Vietoris continuous from $E$ into $P_{k}(C(T, H))$.

Proof. First we will show that if $B \subseteq E$ is compact, then there exists $\Lambda_{B} \in$ $P_{k}(C(T, H))$ such that $S(\lambda) \subseteq \Lambda_{B}$ for all $\lambda \in B$.

Case (i): Assume that condition $H_{c}^{1}(1)$ holds.

Let $\lambda \in B, x(\cdot) \in S(\lambda)$, and let $y_{\lambda}(\cdot) \in C(T, H)$ be the unique strong solution of

$$
-\dot{y}_{\lambda}(t) \in \partial \varphi\left(t, y_{\lambda}(t), \lambda\right) \text { a.e., } \quad y_{\lambda}(0)=x_{0}(\lambda) .
$$

The existence (and of course uniqueness) of $y_{\lambda}(\cdot) \in C(T, H)$ follows from the results of Kenmochi [16] and Yotsutani [32]. As before, exploiting the 
monotonicity of the subdifferential operator and using Lemma A.5, p. 157 of Brezis [6], we get

$$
\left\|x(t)-y_{\lambda}(t)\right\| \leq \int_{0}^{t}\|f(s)\| d s
$$

where $f \in L^{2}(T, H), f(t) \in F(t, x(t), \lambda)$ a.e., and $-\dot{x}(t) \in \partial \varphi(t, x(t), \lambda)+$ $f(t)$ a.e., $x(0)=x_{0}(\lambda)($ recall that $x(\cdot) \in S(\lambda))$. Then we have

$$
\begin{aligned}
\|x(t)\| \leq & \left\|y_{\lambda}\right\|_{C(T, H)}+\int_{0}^{1}\|f(s)\| d s \leq\left\|y_{\lambda}\right\|_{C(T, H)} \\
& +\int_{0}^{t}\left(a_{B}(s)+c_{B}(s)\|x(s)\|\right) d s .
\end{aligned}
$$

Because of hypotheses $H_{c}^{1}(1)$ and $H_{0}$ and the a priori bounds of Yotsutani [32], p. 645, we have that $\sup _{\lambda \in B}\left\|y_{\lambda}\right\|_{C(T, H)}=M_{B}^{\prime}<\infty$. So from Gronwall's inequality, we deduce that there exists $M_{1 B}>0$ such that $\|x(t)\| \leq M_{1 B}$ for all $t \in T$ and all $x(\cdot) \in S(\lambda), \lambda \in B$. Thus without any loss of generality we may assume that $|F(t, x, \lambda)|=\sup \{\|v\|: v \in F(t, x, \lambda)\} \leq a_{B}(t)+c_{B}(t) M_{1 B}=$ $\psi_{B}(t)$ a.e., with $\psi_{B}(\cdot) \in L_{+}^{2}$ (otherwise we can always replace $F(t, x, \lambda)$ by $F\left(t, r_{M_{1 B}}(x), \lambda\right)$, where $r_{M_{1 B}}: H \rightarrow H$ is the $M_{1 B}$-radial retraction). Let $V_{B}=$ $\left\{u \in L^{2}(T, H):\|u(t)\| \leq \psi_{B}(t)\right.$ a.e. $\}$ and set

$$
\Lambda_{B}=p\left(V_{B}, B\right)
$$

where $p: L^{2}(T, H) \times E \rightarrow C(T, H)$ is the map which to each $f \in V_{B}$ and each $z_{0} \in x_{0}(B)=\bigcup_{\lambda \in B} x_{0}(\lambda) \in P_{k}(H)$ (cf. hypothesis $H_{0}$ ), assigns the unique solution of the Cauchy problem $-\dot{x}(t) \in \partial \varphi(t, x(t), \lambda)+f(t)$ a.e., $x(0)=$ $z_{0}=x_{0}(\lambda)$. Our claim is that $\Lambda_{B}$ is compact in $C(T, H)$. To show this, let $x(\cdot) \in \Lambda_{B}$ and $0 \leq t \leq t^{\prime} \leq b$. We have:

$$
\left\|x\left(t^{\prime}\right)-x(t)\right\| \leq \int_{t}^{t^{\prime}}\|\dot{x}(s)\| d s \leq\left(\int_{0}^{b} \chi_{\left[t, t^{\prime}\right]}(s)^{2} d s\right)^{1 / 2}\left(\int_{0}^{b}\|\dot{x}(s)\|^{2} d s\right)^{1 / 2} .
$$

From inequality (7.2), p. 645 of Yotsutani [32], we know that there exists $M_{2 B}>0$ such that

$$
\|\dot{x}(\cdot)\|_{L^{2}(T, H)} \leq M_{2 B}
$$

for all $x \in \Lambda_{B}$. So we have

$$
\left\|x\left(t^{\prime}\right)-x(t)\right\| \leq\left(t^{\prime}-t\right)^{1 / 2} M_{2 B}
$$

$\Rightarrow \Lambda_{B}$ is an equicontinuous subset of $C(T, H)$.

Next let $\Lambda_{B}(t)=\left\{x(t): x(\cdot) \in \Lambda_{B}\right\}, t \in T$. From hypothesis $H_{0}$ and inequality (7.9), p. 645 of Yotsutani [32], we know that there exists $M_{3 B}>0$ such that

$$
\varphi(t, x(t), \lambda) \leq M_{3 B}
$$

for all $x(\cdot) \in \Lambda_{B}$. Hence using hypothesis $H_{c}^{1}(1)$, we have

$$
\Lambda_{B}(t) \subseteq \overline{\bigcup_{\lambda \in B}\left\{y \in H:\|y\|^{2}+\varphi(t, y, \lambda) \leq M_{1 B}^{2}+M_{3 B}=M_{4 B}\right\}} \in P_{k}(H) .
$$

So from the Arzela-Ascoli theorem, we deduce that $\Lambda_{B}$ is relatively compact in $C(T, H)$. We can easily check that $\Lambda_{B}$ is in fact closed in $C(T, H)$. Hence $\Lambda_{B}$ is compact in $C(T, H)$. Finally note that for every $\lambda \in B, S(\lambda) \subseteq \Lambda_{B}$. 
Case (ii): Assume that $H_{c}^{2}(2)$ is valid.

Again by the same a priori estimation as in case (i) above, we get $M_{1 B}>0$ such that $\|x(t)\| \leq M_{1 B}$ for all $t \in T$ and all $x(\cdot) \in S(\lambda), \lambda \in B$. Let $C_{B}=\left\{h \in H:\|h\| \leq M_{1 B}\right\}$ and define

$$
W_{B}(t)=\overline{\operatorname{conv}} F\left(t, C_{B}, B\right) .
$$

Because of hypothesis $H_{c}^{1}(2)$, we know that for all $t \in T, W_{B}(t) \in P_{k c}(H)$. Also because of hypotheses $H(F)_{3}(2)$ and (4), if $\left\{y_{n}\right\}_{n \geq 1}$ is dense in $C_{B}$ and $\left\{\lambda_{m}\right\}_{m \geq 1}$ is dense in $B$, then

$$
W_{B}(t)=\overline{\text { conv }} \bigcup_{\substack{n \geq 1 \\ m \geq 1}} F\left(t, y_{n}, \lambda_{m}\right) \Rightarrow t \rightarrow W_{B}(t) \text { is measurable }
$$

and furthermore $\left|W_{B}(t)\right|=\sup \left\{\|v\|: v \in W_{B}(t)\right\} \leq \psi_{B}(t)$ a.e. with $\psi_{B}(\cdot) \in L_{+}^{2}$ as in case (i). So if we set

$$
S_{B}=\left\{u \in L^{2}(T, H): u(t) \in W_{B}(t) \text { a.e. }\right\}
$$

then we have that $S_{B} \in P_{w k c}\left(L^{2}(T, H)\right)$. Let

$$
\Lambda_{B}=p\left(S_{B}, B\right)
$$

where $p: L^{2}(T, H) \times E \rightarrow C(T, H)$ is the solution map as in case (i). We claim that $\Lambda_{B}$ is compact in $C(T, H)$. As before (cf. case (i)), we can check that $\Lambda_{B}$ is equicontinuous. Also let $\left\{x_{n}\right\}_{n \geq 1} \subseteq \Lambda_{B}$. We have

$$
x_{n}=p\left(f_{n}, \lambda_{n}\right), \quad x_{n}(0)=x_{0}\left(\lambda_{n}\right)
$$

with $f_{n} \in S_{B}, \lambda_{n} \in B$. By passing to a subsequence if necessary, we may assume that $f_{n} \stackrel{w}{\rightarrow} f$ in $L^{2}(T, H)$ with $f \in S_{B}$ and $\lambda_{n} \rightarrow \lambda \in B$ in $E$. Set $x=p(f, \lambda) \in \Lambda_{B}$ with $x(0)=x_{0}(\lambda)$. Since $\Lambda_{B}$ is equicontinuous, a fortiori is weakly equicontinuous and so $\left\{z_{n}(\cdot)=x_{n}(\cdot)-x(\cdot)\right\}_{n \geq 1}$ is weakly equicontinuous in $C(T, H)$. From the a priori estimates we have that for all $n \geq 1$ and all $t \in T$

$$
\begin{aligned}
\left\|z_{n}(t)\right\| & \leq M_{5 B}<\infty \\
\quad \Rightarrow & z_{n}(t) \in \widehat{C}_{B}=\left\{y \in H:\|y\| \leq m_{5 B}\right\} \text { for all } n \geq 1 \text { and all } t \in T .
\end{aligned}
$$

Since $\widehat{C}_{B}$ is weakly compact in $H$, from the Arzela-Ascoli theorem we deduce that $\left\{z_{n}\right\}_{n \geq 1}$ is relatively compact in $C\left(T, \widehat{C}_{B}^{w}\right)$, where $\widehat{C}_{B}^{w}$ denotes the set $\widehat{C}_{B}$ furnished with the relative weak topology of $H$. Recall that $\widehat{C}_{B}^{w}$ is metrizable (see Dunford-Schwartz [12], Theorem 3, p. 434). So from Theorem 8.2 (3), pp. 269-270 of Dugundji [13], we have that $\left\{z_{n}\right\}_{n \geq 1} \subseteq C\left(T, H_{w}\right)$ is relatively sequentially compact in $C\left(T, H_{w}\right) \quad\left(H_{w}\right.$ being the Hilbert space $H$ equipped with the weak topology) and so we may assume that $z_{n} \rightarrow z$ in $C\left(T, H_{w}\right)$. Note that if $y_{n}=p\left(f, \lambda_{n}\right)$ and since $x_{n}=p\left(f_{n}, \lambda_{n}\right)$ and $x=p(f, \lambda)$, we have

$$
\begin{aligned}
\left\|x_{n}(t)-y_{n}(t)\right\|^{2} \leq & 2 \int_{0}^{t}\left(f_{n}(s)-f(s), x_{n}(s)-y_{n}(s)\right) d s \\
= & 2 \int_{0}^{t}\left(f_{n}(s)-f(s), x_{n}(s)-x(s)-z(s)\right) d s \\
& +2 \int_{0}^{t}\left(f_{n}(s)-f(s), x(s)+z(s)-y_{n}(s)\right) d s .
\end{aligned}
$$


Since $z(\cdot) \in C\left(T, H_{w}\right) \Rightarrow z(\cdot) \in L^{\infty}(T, H) \subseteq L^{2}(T, H)$ and so

$$
\int_{0}^{t}\left(f_{n}(s)-f(s), x(s)+z(s)-y_{n}(s)\right) d s \rightarrow 0 \quad \text { as } n \rightarrow \infty .
$$

Also we have $f_{n}(s)-f(s) \in W_{B}(s)-W_{B}(s)=U_{B}(s) \in P_{k c}(H)$ and so from Lemma 3.4 we have that $\int_{0}^{t}\left(f_{n}(s)-f(s), z_{n}(s)-z(s)\right) d s \rightarrow 0$ as $n \rightarrow \infty$. Recall that because of hypothesis $H(\varphi)(3)$ we have $y_{n} \rightarrow x$ in $C(T, H)$. Thus in the limit as $n \rightarrow \infty$, we get

$$
\begin{aligned}
& \left\|x_{n}(t)-x(t)\right\| \rightarrow 0 \quad \text { as } n \rightarrow \infty \text { for all } t \in T \\
& \Rightarrow{\overline{\left\{x_{n}(t)\right\}_{n \geq 1}}} \in P_{k}(H) \quad \text { for all } t \in T \text {. }
\end{aligned}
$$

Since $\Lambda_{B}$ is equicontinuous, from the Arzela-Ascoli theorem we conclude that $\left\{x_{n}(\cdot)\right\}_{n \geq 1}$ is relatively compact in $C(T, H)$ and so $x_{n} \rightarrow x$ in $C(T, H)$, $x \in \Lambda_{B}$. Therefore $\Lambda_{B}$ is compact in $C(T, H)$ and note that $S(\lambda) \subseteq \Lambda_{B}$ for all $\lambda \in B$.

Then from Proposition 3.1 and Remark 1.6 of DeBlasi-Myjak [11], we have that $\lambda \rightarrow S(\lambda)$ is u.s.c. from $E$ into $P_{k}(C(T, H))$. On the other hand from Proposition 3.2 we know that $\lambda \rightarrow S(\lambda)$ is l.s.c. from $E$ into $P_{k}(C(T, H))$. So we conclude that $\lambda \rightarrow S(\lambda)$ is Vietoris continuous from $E$ into $P_{k}(C(T, H))$.

On $P_{k}(C(T, H))$ the Vietoris and Hausdorff metric hyperspace topologies coincide (see Klein-Thompson [17], Corollary 4.2.3, p. 41). So from Theorem 3.5 we immediately get the following result:

Theorem 3.6. If hypotheses $H(\varphi), H(F)_{3}, H_{c}^{1}$, and $H_{0}$ hold, then the multifunction $\lambda \rightarrow S(\lambda)$ is h-continuous from $E$ into $P_{k}(C(T, H))$.

\section{SENSITIVITY ANALYSIS OF OPTIMAL CONTROL PROBLEMS}

In this section, we will use the continuous dependence results of Section 3 to conduct a sensitivity analysis for a class of nonlinear, infinite dimensional optimal control problems. Such a sensitivity analysis (often called in the literature "variational analysis") is important because it generates useful information about the tolerances that are permitted in the specification of mathematical models, it suggests ways to analyze parametric problems and establish various kinds of continuous dependence results, it is helpful in problems of identification of parameters, and it can also lead to robust computational schemes for the numerical treatment of the problem.

So let $Y$ be a separable reflexive Banach space, modeling the control space. The problem under consideration is the following:

$$
\left\{\begin{array}{c}
J(x, u, \lambda)=\int_{0}^{b} L(t, x(t), u(t), \lambda) d t \rightarrow \inf =m(\lambda) \\
\text { s.t. }-\dot{x}(t) \in \partial \varphi(t, x(t), \lambda)+g(t, x(t), \lambda)+B(t, \lambda) u(t) \text { a.e. } \\
x(0)=x_{0}(\lambda) \\
u(t) \in U(t, \lambda) \text { a.e. }
\end{array}\right\}
$$

Let $P(\lambda)=\left\{[x, u] \in C(T, H) \times L^{2}(T, Y):[x, u]\right.$ is an optimal pair for (2)\}. Our goal is to investigate the continuity properties of the value function $\lambda \rightarrow m(\lambda)$ and those of the multifunction $\lambda \rightarrow P(\lambda)$. 
We will need the following hypotheses on the data:

$H(g): g: T \times H \times E \rightarrow H$ is a map such that

(1) $t \rightarrow g(t, x, \lambda)$ is measurable,

(2) $\|g(t, x, \lambda)-g(t, y, \lambda)\| \leq k_{B}(t)\|x-y\|$ a.e. for all $\lambda \in B \subseteq E$ compact with $k_{B}(\cdot) \in L_{+}^{1}$

(3) $\|g(t, x, \lambda)\| \leq a_{B}^{1}(t)+c_{B}^{1}(t)\|x\|$ a.e. for all $\lambda \in B \subseteq E$ compact and with $a_{B}^{1}, c_{B}^{1} \in L_{+}^{2}$,

(4) $\lambda \rightarrow g(t, x, \lambda)$ is continuous.

$H(B): B: T \times E \rightarrow \mathscr{L}(Y, H)$ is a map such that

(1) for all $\lambda \in E, B(\cdot, \lambda) \in L^{\infty}(T, \mathscr{L}(Y, H))$ and for all $B \subseteq E$ compact $\sup _{\lambda \in B}\|B(\cdot, \lambda)\|_{L^{\infty}(T, \mathscr{L}(Y, H))}=M_{B}<\infty$,

(2) $\lambda \rightarrow B(t, \lambda) u$ and $\lambda \rightarrow B(t, \lambda)^{*} h$ are both continuous for every $(u, h)$ $\in Y \times H$.

$H(U): U: T \times E \rightarrow P_{w k c}(Y)$ is a multifunction such that

(1) $t \rightarrow U(t, \lambda)$ is measurable,

(2) $\lambda \rightarrow U(t, \lambda)$ is $h$-continuous,

(3) $|U(t, \lambda)|=\sup \left\{\|u\|_{Y}: u \in U(t, \lambda)\right\} \leq a_{B}^{2}(t)$ a.e. for all $\lambda \in B \subseteq E$ compact and with $a_{B}^{2}(\cdot) \in L_{+}^{2}$.

$H(L): L: T \times H \times Y \times E \rightarrow \mathbb{R}$ is an integrand such that

(1) $t \rightarrow L(t, x, u, \lambda)$ is measurable,

(2) $(x, u, \lambda) \rightarrow L(t, x, u, \lambda)$ is continuous,

(3) $u \rightarrow L(t, x, u, \lambda)$ is convex,

(4) $r(t)-M_{B}\left(\|x\|+\|u\|_{Y}\right) \leq L(t, x, u, \lambda)$ a.e. for all $\lambda \in B \subseteq E$ compact with $r(\cdot) \in L_{+}^{1}$ and $M_{B}>0$ and furthermore if $x_{n} \rightarrow x$ in $L^{2}(T, H)$, $u_{n} \rightarrow u$ in $L^{2}(T, Y)$ and $\lambda_{n} \rightarrow \lambda$ in $E$, then $\left.\left\{L(\cdot), x_{n}(\cdot), u_{n}(\cdot), \lambda_{n}\right)\right\}_{n \geq 1}$ is uniformly integrable.

Finally hypothesis $H_{c}^{1}$ takes the following form:

$H_{c}^{2}$ : One of the following two conditions holds:

(1) the same as $H_{c}^{1}$,

(2) for every $t \in T$, every $B \subseteq E$ compact, and every $K \subseteq H$ bounded, we have $\overline{\bigcup_{\lambda \in B} g(t, K, \lambda)}$ and $\bigcup_{\lambda \in B} B(t, \lambda) U(t, \lambda)$ are both compact in $H$; i.e. the families $\{g(t, \cdot, \lambda)\}_{\lambda \in B}$ and $\{B(t, \lambda)(\cdot)\}_{\lambda \in B}$ are collectively compact.

With these hypotheses we can prove the following result concerning the value function $\lambda \rightarrow m(\lambda)$ of problem (2).

Theorem 4.1. If hypotheses $H(\varphi), H(g), H(B), H(U), H(L), H_{c}^{2}$, and $H_{0}$ hold, then $\lambda \rightarrow m(\lambda)$ is continuous from $E$ into $\mathbb{R}$.

Proof. First we will show that the dynamics of (2) can be expressed by an equivalent evolution inclusion of the form (1) (see Section 1) (deparametrized or control-free dynamics). Indeed let $F: T \times H \times E \rightarrow P_{w k c}(H)$ be defined by

$$
F(t, x, \lambda)=g(t, x, \lambda)+B(t, \lambda) U(t, \lambda) .
$$


Fix $(x, \lambda) \in H \times E$ and let $u_{n}: T \rightarrow Y, n \geq 1$, be measurable functions

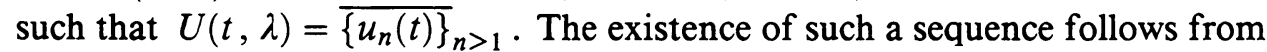
hypothesis $H(U)(1)$ and Theorem 4.2 of Wagner [30]. Then $F(t, x, \lambda)=$ $\left\{g(t, x, \lambda)+B(t, \lambda) u_{n}(t)\right\}_{n \geq 1}$, which implies that $t \rightarrow F(t, x, \lambda)$ is a measurable multifunction.

Also note that for all $\lambda \in B \subseteq E$ compact, we have

$$
h(F(t, x, \lambda), F(t, y, \lambda)) \leq\|g(t, x, \lambda)-g(t, y, \lambda)\| \leq k_{B}(t)\|x-y\| \text { a.e. }
$$

(cf. hypothesis $H(g)(2)$ ). Furthermore using hypotheses $H(g)(3), H(B)(1)$, and $H(U)(3)$ we get for all $\lambda \in B \subseteq E$ compact:

$$
|F(t, x, \lambda)|=\sup \{\|v\|: v \in F(t, x, \lambda)\} \leq a_{B}(t)+c_{B}(t)\|x\| \text { a.e. }
$$

with $a_{B}(\cdot)=a_{B}^{1}(\cdot)+a_{B}^{2}(\cdot) \in L_{+}^{2}$ and $c_{B}(\cdot)=c_{B}^{1}(\cdot)+c_{B}^{2}(\cdot) \in L_{+}^{2}$. get

Finally if $\lambda_{n} \rightarrow \lambda$ in $E$, then using hypotheses $H(g)(4)$ and $H(U)(2)$, we

$$
\begin{aligned}
& \quad h\left(F\left(t, x, \lambda_{n}\right), F(t, x, \lambda)\right) \\
& \quad \leq\left\|g\left(t, x, \lambda_{n}\right)-g(t, x, \lambda)\right\|+M_{B} h\left(U\left(t, \lambda_{n}\right), U(t, \lambda)\right) \rightarrow 0 \text { as } n \rightarrow \infty \\
& \Rightarrow \lambda \rightarrow F(t, x, \lambda) \text { is } h \text {-continuous. }
\end{aligned}
$$

Hence $F(t, x, \lambda)$ satisfies hypothesis $H(F)_{3}$. A straightforward application of Aumann's selection theorem (see Wagner [30], Theorem 5.10), reveals that the dynamics of problem (2) are equivalent to the following subdifferential evolution inclusion:

$$
\left\{\begin{array}{c}
-\dot{x}(t) \in \partial \varphi(t, x(t), \lambda)+F(t, x(t), \lambda) \text { a.e. } \\
x(0)=x_{0}(\lambda)
\end{array}\right\} .
$$

We know (see Section 3), that for every $\lambda \in E$ the solution set of the above inclusion is compact in $C(T, H)$. So if $R(\lambda)=\{[x, u] \in C(T, H) \times$ $L^{2}(T, Y):[x, u]$ is a state-control pair satisfying the constraints of problem (2)\} (i.e. $R(\lambda) \subseteq C(T, H) \times L^{2}(T, Y)$ is the set of admissible states and controls), then we easily see that $R(\lambda)$ is compact in $C(T, H) \times L^{2}(T, Y)_{w}$, with $L^{2}(T, Y)_{w}$ the Lebesgue-Bochner space $L^{2}(T, Y)$ equipped with the weak topology. Also from hypothesis $H(L)$ and Theorem 2.1 of Balder [4], we have that $(x, u) \rightarrow J(x, u, \lambda)$ is 1.s.c. from $L^{1}(T, H) \times L^{1}(T, Y)_{w}$ into $\mathbb{R}$. Therefore we get that for every $\lambda \in E$, the corresponding optimal control problem (2) has a solution; i.e. there exists an admissible state-control pair $[x, u] \in R(\lambda)$ such that $J(x, u, \lambda)=m(\lambda)$ (so $[x, u] \in P(\lambda)$ ).

Next, let $\lambda_{n} \rightarrow \lambda$ and let $\left[x_{n}, u_{n}\right] \in R\left(\lambda_{n}\right)$ such that $J\left(x_{n}, u_{n}, \lambda_{n}\right)=$ $m\left(\lambda_{n}\right), n \geq 1$.

From the proof of Theorem 3.5 and hypothesis $H(U)$, we know that by passing to a subsequence if necessary, we may assume that $x_{n} \rightarrow x$ in $C(T, H)$, $\dot{x}_{n} \stackrel{w}{\rightarrow} \dot{x}$ in $L^{2}(T, H)$, and $u_{n} \stackrel{w}{\rightarrow} u$ in $L^{2}(T, Y)$. Let $\hat{g}: L^{2}(T, H) \times E \rightarrow$ $L^{2}(T, H)$ and $\widehat{B}: E \rightarrow L^{\infty}(T, \mathscr{L}(Y, H))$ be the Nemitsky (superposition) operators corresponding to the functions $g(\cdot, x(\cdot), \lambda)$ and $B(\cdot, \lambda)$; i.e. $\hat{g}(x, \lambda)(\cdot)$ $=g(\cdot, x(\cdot), \lambda)$ and $\widehat{B}(\lambda)(\cdot)=B(\cdot, \lambda)$. Then from hypotheses $H(g)$ and $H(B)$, we have that $\hat{g}\left(x_{n}, \lambda_{n}\right) \rightarrow \hat{g}(x, \lambda)$ in $L^{2}(T, H)$ and $\widehat{B}\left(\lambda_{n}\right) u_{n} \stackrel{w}{\rightarrow} \widehat{B}(\lambda) u$ in $L^{2}(T, H)$. Note that

$$
\left[x_{n},-\dot{x}_{n}-\hat{g}\left(x_{n}, \lambda_{n}\right)-\widehat{B}\left(\lambda_{n}\right) u_{n}\right] \in \operatorname{Gr} \partial \Phi\left(\cdot, \lambda_{n}\right)
$$


and as in the proof of Proposition 3.1, passing to the limit as $n \rightarrow \infty$, and using hypothesis $H(\varphi)$, we get

$$
\begin{gathered}
{[x,-\dot{x}-\hat{g}(x, \lambda)-\widehat{B}(\lambda) u] \in \operatorname{Gr} \partial \Phi(\cdot, \lambda)} \\
\Rightarrow-\dot{x}(t) \in \partial \varphi(t, x(t), \lambda)+g(t, x(t), \lambda)+B(t, \lambda) u(t) \text { a.e. } \\
x(0)=x_{0}(\lambda) .
\end{gathered}
$$

Also note that for every $q \in L^{2}\left(T, Y^{*}\right)=L^{2}(T, Y)^{*}$, we have

$$
\left\langle u_{n}, q\right\rangle=\int_{0}^{b}\left(u_{n}(t), q(t)\right)_{Y, Y^{*}} d t \leq \int_{0}^{b} \sigma\left(q(t), U\left(t, \lambda_{n}\right)\right) d t
$$

where $\sigma\left(q(t), U\left(t, \lambda_{n}\right)\right)=\sup \left\{(q(t), u)_{Y^{*}, Y}: u \in U\left(t, \lambda_{n}\right)\right\}$ (the support function of $\left.U\left(t, \lambda_{n}\right)\right)$. From hypothesis $H(U)(2)$, we know that $\sigma\left(q(t), U\left(t, \lambda_{n}\right)\right)$ $\rightarrow \sigma(q(t), U(t, \lambda))$. So through the dominated convergence theorem, in the limit we get

$$
\begin{aligned}
\langle u, q\rangle & =\int_{0}^{b}(u(t), q(t))_{Y, Y^{*}} d t \leq \int_{0}^{b} \sigma(q(t), U(t, \lambda)) d t \text { for all } q \in L^{2}\left(T, Y^{*}\right) \\
& \Rightarrow u(t) \in U(t, \lambda) \text { a.e. }
\end{aligned}
$$

Thus finally we have $[x, u] \in R(\lambda)$. Applying Theorem 2.1 of Balder [4], we get

$$
\begin{aligned}
J(x, u, \lambda) & \leq \underline{\lim } J\left(x_{n}, u_{n}, \lambda_{n}\right)=\underline{\lim } m\left(\lambda_{n}\right) \\
& \Rightarrow m(\lambda) \leq \underline{\lim } m\left(\lambda_{n}\right) .
\end{aligned}
$$

Next let $[x, u] \in R(\lambda)$ such that $J(x, u, \lambda)=m(\lambda)$. Then because of hypothesis $H_{c}^{2}$, we can apply Proposition 3.2 and find admissible states $x_{n}(\cdot) \in$ $C(T, H), n \geq 1$, corresponding to the parameter $\lambda_{n} \in E$ such that $x_{n} \rightarrow x$ in $C(T, H)$. Also because of hypothesis $H(U)$ and Theorem 4.5 of [24], we know that we can find control functions $u_{n} \in S_{U\left(\cdot, \lambda_{n}\right)}^{2}$ such that $u_{n} \stackrel{s}{\rightarrow} u$ in $L^{2}(T, Y)$. Let $z_{n}=p\left(\hat{g}\left(x_{n}, \lambda_{n}\right)+\widehat{B}\left(\lambda_{n}\right) u, \lambda_{n}\right)$ where as before $p: L^{2}(T, H) \times$ $E \rightarrow C(T, H)$ is the solution map. Since $\hat{g}\left(x_{n}, \lambda_{n}\right)+\widehat{B}\left(\lambda_{n}\right) u \rightarrow \hat{g}(x, \lambda)+\widehat{B}(\lambda) u$ in $L^{2}(T, H)$ and $p(\cdot, \cdot)$ is continuous, we have that

$$
z_{n} \rightarrow p(\hat{g}(x, \lambda)+\widehat{B}(\lambda) u, \lambda)=x \text { in } C(T, H) .
$$

Let $y_{n} \in C(T, H)$ be the unique admissible state of problem (2) generated by $u_{n}(\cdot)$ and corresponding to the parameter $\lambda_{n} \in E$; i.e. $\left[y_{n}, u_{n}\right] \in R\left(\lambda_{n}\right), n \geq 1$. From the proof of Theorem 3.5 we know that $\left\{y_{n}(\cdot)\right\}_{n \geq 1} \subseteq \Lambda_{B} \in P_{k}(C(T, H))$ with $B=\left\{\lambda_{n}, \lambda\right\}_{n \geq 1}$. So by passing to a subsequence if necessary, we may assume that $y_{n} \rightarrow y$ in $C(T, H)$. Also exploiting the monotonicity of the subdifferential operator, we have

$$
\begin{aligned}
\left\|y_{n}(t)-z_{n}(t)\right\| \leq & \int_{0}^{t}\left\|g\left(s, y_{n}(s), \lambda_{n}\right)-g\left(s, x_{n}(s), \lambda_{n}\right)\right\| d s \\
& +\int_{0}^{t}\left\|B\left(s, \lambda_{n}\right)\left(u_{n}(s)-u(s)\right)\right\| d s \\
\leq & \int_{0}^{t} k_{B}(s)\left\|y_{n}(s)-x_{n}(s)\right\| d s+\int_{0}^{t} M_{B}\left\|u_{n}(s)-u(s)\right\| d s .
\end{aligned}
$$


Passing to the limit as $n \rightarrow \infty$, we get

$$
\begin{aligned}
\|y(t)-x(t)\| & \leq \int_{0}^{t} k_{B}(s)\|y(s)-x(s)\| d s \quad \text { for all } t \in T \\
& \Rightarrow x=y .
\end{aligned}
$$

Then from hypothesis $H(L)(4)$ and the extended dominated convergence theorem, we have that

$$
J\left(y_{n}, u_{n}, \lambda_{n}\right) \rightarrow J(x, u, \lambda)=m(\lambda) \Rightarrow \varlimsup \lim m\left(\lambda_{n}\right) \leq m(\lambda) .
$$

From inequalities (3) and (4) above we get that

$$
m\left(\lambda_{n}\right) \rightarrow m(\lambda) \Rightarrow \lambda \rightarrow m(\lambda) \text { is continuous. }
$$

Using this continuity result, we can prove the following property of the optimal pairs multifunction $\lambda \rightarrow P(\lambda)$.

Theorem 4.2. If hypotheses $H(\varphi), H(g), H(B), H(U), H(L), H_{c}^{2}$, and $H_{0}$ hold, then the multifunction $\lambda \rightarrow P(\lambda)$ is u.s.c. from $E$ into

$$
P_{k}\left(C(T, H) \times L^{2}(T, Y)_{w}\right) .
$$

Proof. Note that for every $B \subseteq E$ compact

$$
\widehat{\bigcup}_{\lambda \in B} S_{U(\cdot, \lambda)}^{2} \subseteq V=\left\{u \in L^{2}(T, H):\|u\|_{L^{2}(T, Y)} \leq\left\|a_{B}^{2}\right\|_{2}\right\}
$$

and the latter equipped with the relative weak $L^{2}(T, Y)$ topology is compact metrizable. Hence since for every $B \subseteq E$ compact, $\overline{P(B)} \subseteq \Lambda_{B} \times V$ for some $\Lambda_{B} \in P_{k}(C(T, H)$ ) (cf. the proof of Theorem 3.5), to establish the desired upper semicontinuity of $P(\cdot)$, it is enough to show that if $\lambda_{n} \rightarrow \lambda$ in $E,\left[x_{n}, u_{n}\right] \rightarrow$ $[x, u], C(T, H) \times L^{2}(T, Y)_{w}$, and $\left[x_{n}, u_{n}\right] \in P\left(\lambda_{n}\right), n \geq 1$, then $[x, u] \in$ $P(\lambda)$ (see DeBlasi-Myjak [11], Remark 1.6). But by hypothesis

$$
J\left(x_{n}, u_{n}, \lambda_{n}\right)=m\left(\lambda_{n}\right), \quad n \geq 1 .
$$

Also from Theorem 2.1 of Balder [4], we know that

$$
J(x, u, \lambda) \leq \underline{\lim } J\left(x_{n}, u_{n}, \lambda_{n}\right)
$$

while from Theorem 4.1 we have that $m\left(\lambda_{n}\right) \rightarrow m(\lambda)$. Since $[x, u] \in R(\lambda)$ (see the proof of Theorem 4.1), we conclude that $J(x, u, \lambda)=m(\lambda)$; i.e. $[x, u] \in$ $P(\lambda)$.

In fact our general framework of subdifferential evolution inclusions allows us to treat a class of nonlinear infinite dimensional optimal control problems with a priori feedback (state dependent control constraints). More precisely we consider the following problem:

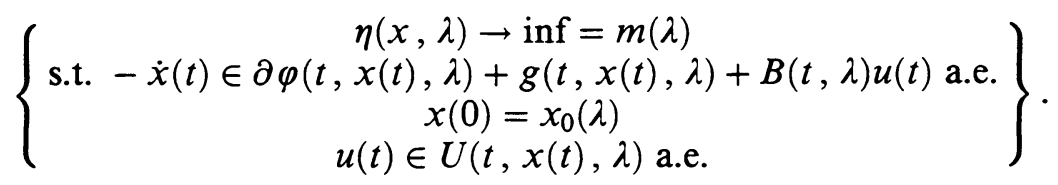

We will need the following hypotheses on the multifunction $U(t, x, \lambda)$ and the cost criterion $\eta(x, \lambda)$. 
$H(U)_{1}: U: T \times H \times E \rightarrow P_{w k c}(Y)$ is a multifunction such that

(1) $t \rightarrow U(t, x, \lambda)$ is measurable,

(2) $h(U(t, x, \lambda), U(t, y, \lambda)) \leq k_{B}^{\prime}(t)\|x-y\|$ a.e. for all $\lambda \in B \subseteq E$ compact and with $k_{B}^{\prime}(\cdot) \in L_{+}^{1}$.

(3) $|U(t, x, \lambda)|=\sup \{\|u\|: u \in U(t, x, \lambda)\} \leq a_{B}^{2}(t)+c_{B}^{2}(t)\|x\|$ a.e. for all $\lambda \in B \subseteq E$ compact and with $a_{B}^{2}, c_{B}^{2} \in L_{+}^{2}$,

(4) $\lambda \rightarrow U(t, x, \lambda)$ is $h$-continuous.

$H(\eta): \eta: C(T, H) \times E \rightarrow \mathbb{R}$ is a continuous map.

Then we have the following sensitivity result for problem (5):

Theorem 4.3. If hypotheses $H(\varphi), H(g), H(B), H(U)_{1}, H(\eta), H_{c}^{2}$, and $H_{0}$ hold, then $\lambda \rightarrow m(\lambda)$ is continuous from $E$ into $\mathbb{R}$ and $\lambda \rightarrow P(\lambda)$ is u.s.c. from E into

$$
P_{k}\left(C(T, H) \times L^{2}(T, Y)_{w}\right) .
$$

Proof. As in the proof of Theorem 4.1, we can check that $t \rightarrow F(t, x, \lambda)$ is measurable, $|F(t, x, \lambda)|=\sup \{\|v\|: v \in F(t, x, \lambda)\} \leq \hat{a}_{B}(t)+\hat{c}_{B}(t)\|x\|$ a.e. for all $\lambda \in B \subseteq E$, with $\hat{a}_{B}, \hat{c}_{B} \in L_{+}^{2}$ and $\lambda \rightarrow F(t, x, \lambda)$ is $h$-continuous.

Also note that for all $\lambda \in B \subseteq E$ compact, we have

$$
\begin{aligned}
& h(F(t, x, \lambda), F(t, y, \lambda)) \\
& \quad \leq\|g(t, x, \lambda)-g(t, y, \lambda)\|+M_{B} h(U(t, x, \lambda), U(t, y, \lambda)) \\
& \quad \leq k_{B}(t)\|x-y\|+M_{B} k l_{B}^{\prime}(t)\|x-y\|=\hat{k}_{B}(t)\|x-y\| \text { a.e. }
\end{aligned}
$$

with $\hat{k}_{B}(\cdot)=k_{B}(\cdot)+M_{B} k_{B}^{\prime}(\cdot) \in L_{+}^{1}$. So we have satisfied hypothesis $H(F)_{3}$. Then through Aumann's selection theorem (see Wagner [30], Theorem 5.10), we can check that problem (5) is equivalent to the following deparametrized (control-free) system:

$$
\left\{\begin{array}{c}
\eta(x, \lambda) \rightarrow \inf =m(\lambda) \\
\text { s.t. }-\dot{x}(t) \in \partial \varphi(t, x(t), \lambda)+F(t, x(t), \lambda) \text { a.e. } \\
x(0)=x_{0}(\lambda)
\end{array}\right\} .
$$

We know that the set of admissible states of (6) is compact (cf. Section 3 ). So because of the continuity of $\eta(\cdot, \cdot)$ (cf. hypothesis $H(\eta)$ ), we see that for every $\lambda \in E$, problem (6) (and so equivalently problem (5)) has a solution.

Let $\lambda_{n} \rightarrow \lambda$ and choose $x_{n} \in C(T, H)$ an admissible state corresponding to the parameter $\lambda_{n}$, for which $\eta\left(x_{n}, \lambda_{n}\right)=m\left(\lambda_{n}\right)$. Recall (see the proof of Theorem 3.5) that the sequence $\left\{x_{n}(\cdot)\right\}_{n \geq 1}$ is relatively compact in $C(T, H)$. So by passing to a subsequence if necessary, we may assume that $x_{n} \rightarrow x$ in $C(T, H)$. From Theorem 3.5 we know that $x(\cdot)$ is an admissible state for the parameter $\lambda \in E$. Then $\eta\left(x_{n}, \lambda_{n}\right)=m\left(\lambda_{n}\right) \rightarrow \eta(x, \lambda) \geq m(\lambda)$ and so

$$
m(\lambda) \leq \underline{\lim } m\left(\lambda_{n}\right) \text {. }
$$

Next let $x(\cdot) \in C(T, H)$ be admissible for $\lambda \in E$ and $\eta(x, \lambda)=m(\lambda)$. Once again invoking Theorem 3.5, we can find $x_{n}(\cdot) \in C(T, H)$ admissible for $\lambda_{n} \in E$ such that $x_{n} \rightarrow x$ in $C(T, H)$. So $\eta\left(x_{n}, \lambda_{n}\right) \rightarrow \eta(x, \lambda)=m(\lambda)$. Hence

$$
\varlimsup m\left(\lambda_{n}\right) \leq m(\lambda) .
$$

From (7) and (8), we deduce that $m\left(\lambda_{n}\right) \rightarrow m(\lambda)$; i.e. $m(\cdot)$ is continuous. 
Finally the upper semicontinuity of $P(\cdot)$ follows from the continuity of $m(\cdot)$ as in the proof of Theorem 4.2.

\section{EXAMPLES}

In this section we present some examples illustrating the applicability of our work.

(i) Our first example deals with a parametrized family of nonlinear parabolic variational inequalities with unilateral constraints (obstacle problem).

So let $Z$ be a bounded domain in $\mathbb{R}^{N}$ with smooth boundary $\Gamma=\partial Z$ and $2 \leq p<\infty$ with $N \leq \frac{2 p}{p-2}$ if $p \neq 2$. Let $g: T \times Z \rightarrow \mathbb{R}$ and for every $t \in T$ define

$$
K(t)=\left\{h \in W^{1, p}(Z): h(z) \geq g(t, z) \text { a.e. on } Z\right\} .
$$

Clearly this is a closed and convex subset of $W^{1, p}(Z)$. We consider the problem of finding a function $x(t, z)$ defined on $T \times Z$ with values in $\mathbb{R}$ satisfying (here $\left.D_{k}=\frac{\partial}{\partial z_{k}}, k=1, \ldots, N\right)$ :

$$
\left\{\begin{array}{c}
\frac{\partial x}{\partial t}-\sum_{k=1}^{N} D_{k}\left(a(z, \lambda)\left|D_{k} x\right|^{p-2} D_{k} x\right) \geq f(t, z) \text { on } T \times Z \\
x(t, z) \geq g(t, z) \text { a.e. on } Z \text { for all } t \in T \\
\left(\frac{\partial x}{\partial t}-\sum_{k=1}^{N} D_{k}\left(a(z, \lambda)\left|D_{k} x\right|^{p-2} D_{k} x\right)\right)(x(t, z)-g(t, z))=0 \text { on } T \times Z \\
x(0, z)=x_{0}(z, \lambda) \text { a.e. on } Z \\
\xi_{1}(t, z, x(t, z), \lambda) \leq f(t, z) \leq \xi_{2}(t, z, x(t, z), \lambda) \text { a.e. on } T \times Z
\end{array}\right\} .
$$

We will need the following hypotheses on the data of (9).

$H(g)_{1}: g: T \times Z \rightarrow \mathbb{R}$ is a function such that

(1) for every $t \in T, g(t, \cdot) \in W^{1, \infty}(Z)$,

(2) there exist $0<c_{1}<c_{2}$ such that $c_{1} \leq g(t, z) \leq c_{2}$ and $\|D g(t, z)\|_{N} \leq$ $c_{2}$ for all $t \in T$ and almost all $z \in Z$ (here $\|\cdot\|_{N}$ denotes the $l^{p}$-norm on $\mathbb{R}^{N}$ ),

(3) there exist $r_{1} \in W^{1,2}(T)$ and $r_{2}: T \rightarrow \mathbb{R}$ of bounded variation such that for all $t, s \in T \backslash N, \mu(N)=0(\mu(\cdot)$ being the Lebesgue measure on $T)$, we have

$$
|g(t, z)-g(s, z)| \leq\left|r_{1}(t)-r_{1}(s)\right| \text { a.e. on } Z
$$

and $\|D g(t, \cdot)-D g(s, \cdot)\|_{L^{2}(Z)} \leq\left|r_{2}(t)-r_{2}(s)\right|$.

$H(a): 0<m_{1 B} \leq a(z, \lambda) \leq m_{2 B}$ a.e. on $Z$ for every $\lambda \in B \subseteq E$ compact and if $\lambda_{n} \rightarrow \lambda$ in $E$ then $a\left(z, \lambda_{n}\right) \rightarrow a(z, \lambda)$ a.e. on $Z$.

$H(\xi): \xi_{i}: T \times Z \times \mathbb{R} \times E \rightarrow \mathbb{R}, i=1,2$, are functions such that $\xi_{1} \leq \xi_{2}$ and for $i=1,2$,

(1) $(t, z) \rightarrow \xi_{i}(t, z, x, \lambda)$ is measurable,

(2) $\left|\xi_{i}(t, z, x, \lambda)-\xi_{i}(t, z, y, \lambda)\right| \leq k_{B}(t, z)|x-y|$ a.e. for all $\lambda \in B \subseteq E$ compact and with $k_{B} \in L^{1}\left(T, L^{2}(Z)\right)$,

(3) $\left|\xi_{i}(t, z, x, \lambda)\right| \leq a_{B}(t, z)+c_{B}(t, z)|x|$ a.e. for all $\lambda \in B \subseteq E$ compact and with $a_{B}, c_{B} \in L^{2}(T \times Z)$,

(4) $\lambda \rightarrow \xi_{i}(t, z, x, \lambda)$ is continuous. 
$H_{0}^{\prime}: \lambda \rightarrow x_{0}(\cdot, \lambda)$ is continuous from $E$ into $L^{2}(Z)$ and for all $\lambda \in E, x_{0}(\cdot, \lambda)$ $\in K(0)$.

Let $S(\lambda) \subseteq C\left(T, L^{2}(Z)\right)$ be the set of solutions of (9). We can prove the following result about the multifunction $\lambda \rightarrow S(\lambda)$.

Theorem 5.1. If hypotheses $H(g)_{1}, H(a), H(\xi)$, and $H_{0}^{\prime}$ hold, then $\lambda \rightarrow S(\lambda)$ is a Vietoris and h-continuous multifunction from $E$ into $P_{k}\left(C\left(T, L^{2}(Z)\right)\right)$. Proof. Let $H=L^{2}(Z)$ and let $\varphi: T \times H \times E \rightarrow \overline{\mathbb{R}}=\mathbb{R} \cup\{+\infty\}$ be defined by

$$
\varphi(t, x, \lambda)= \begin{cases}\frac{1}{p} \int_{Z} a(z, \lambda)\|D x(z)\|_{N}^{p} d z & \text { if } x \in K(t), \\ +\infty & \text { otherwise, }\end{cases}
$$

where $D=\left(D_{k}\right)_{k=1}^{N}$, the gradient operator. It is easy to check that for every $(t, \lambda) \in T \times E, \varphi(t, \cdot, \lambda) \in \Gamma_{0}(H)$.

Next let $s, t \in T$ and $x(\cdot) \in K(t)$. Define

$$
y(z)=\frac{g(s, z)}{g(t, z)} x(z) .
$$

Clearly, since $x(\cdot) \in K(t)$, we have that $y(\cdot) \in K(s)$. In addition we have

$$
\begin{aligned}
\int_{Z}|y(z)-x(z)|^{2} d z & =\int_{Z}\left|\frac{g(s, z)}{g(t, z)}-1\right|^{2}|x(z)|^{2} d z \\
& \leq \int_{Z} \frac{|g(s, z)-g(t, z)|^{2}}{c_{1}^{2}}|x(z)|^{2} d z \\
& \leq \frac{1}{c_{1}^{2}}\left|r_{1}(s)-r_{2}(t)\right|^{2}|x(z)|^{2} d z .
\end{aligned}
$$

So if $\|x\|_{2} \leq r$, then $\|x-y\|_{L^{2}(Z)} \leq c_{3}\left|r_{1}(t)-r_{1}(s)\right|^{2} r$, with $c_{3}=1 / c_{1}^{2}$ and so we have satisfied the first inequality in hypothesis $H(\varphi)(2)$ with $g_{r}(t)=$ $c_{3} r_{1}(t) r$.

Next we have

$$
\begin{aligned}
& \|D y(z)\|_{N}^{p}-\|D x(z)\|_{N}^{p} \\
& \quad=\left\|\frac{g(s, z)}{g(t, z)} D x(z)+\frac{g(t, z) D g(s, z)-g(s, z) D g(t, z)}{g(t, z)^{2}} x(z)\right\|_{N}^{p}-\|D x(z)\|_{N}^{p} .
\end{aligned}
$$

Then we can find $c_{4}>0$ (independent of $t, s, x$ ) large enough (i.e. $c_{4}>$ $2^{p-1}$ and $\left.\left(c_{2} / c_{1}\right)^{p} \leq\left(c_{4}-2^{p-1}\right) /\left(c_{4}-1\right)\right)$ such that

$$
\begin{aligned}
& \|D y(z)\|_{N}^{p}-\|D x(z)\|_{N}^{p} \\
& \quad \leq c_{4}\left(\left|\frac{g(s, z)}{g(t, z)}\right|^{p}-1\right)\|D x(z)\|_{N}^{p}+c_{4} \frac{\|g(t, z) D g(s, z)-g(s, z) D g(t, z)\|_{N}^{p}}{c_{1}^{p}} .
\end{aligned}
$$

Hence by elementary algebraic calculations and using hypothesis $H(g)_{1}$, we get that there exists $c_{5}>0$ (independent of $\left.t, s, x\right)$ such that

$$
|\varphi(t, x, \lambda)-\varphi(s, y, \lambda)| \leq c_{5}\left(\left|r_{1}(t)-r_{1}(s)\right|+\left|r_{2}(t)-r_{2}(s)\right|\right)(1+\varphi(t, x, \lambda)) .
$$

So we have satisfied hypotheses $H(\varphi)(1)$ and (2) with $\alpha=\frac{1}{p} \in\left[0, \frac{1}{2}\right]$, hence $\beta=2, g_{r}=c_{3} r_{1}(\cdot) r$, and $h_{r}=c_{5}\left(\int_{0}^{t}\left|\dot{r}_{1}(s)\right| d s+r_{2}(t)\right)$. 
Furthermore we claim that if $\lambda_{n} \rightarrow \lambda$ in $E$, then $\varphi\left(t, \cdot, \lambda_{n}\right) \stackrel{M}{\rightarrow} \varphi(t, \cdot, \lambda)$ in $L^{2}(Z)$. Indeed let $x_{n} \stackrel{w}{\rightarrow} x$ in $L^{2}(Z)$. We need to show that $\varphi(t, x, \lambda) \leq$

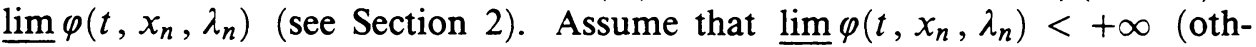
erwise the inequality is automatically valid). Take a subsequence such that $\lim \varphi\left(t, x_{n_{k}}, \lambda_{n_{k}}\right)=\underline{\lim } \varphi\left(t, x_{n}, \lambda_{n}\right)<\infty$. Then from the definition of $\varphi$, and using the Sobolev embedding theorem (recall $N \leq \frac{2 p}{p-2}, p \neq 2$ ), we can see that by passing to a subsequence if necessary $x_{n_{k}} \stackrel{w}{\rightarrow} x \in K(t)$ in $W^{1, p}(Z)$. But from hypothesis $H(a)$ and Theorem 5.14, p. 51 of Dal Maso [9], we have that

$$
\begin{aligned}
\int_{Z} a(z, \lambda)\|D x(z)\|_{N}^{p} d z & \leq \underline{\lim } \int_{Z} a\left(z, \lambda_{n_{k}}\right)\left\|D x_{n_{k}}(z)\right\|_{N}^{p} d z \\
& \Rightarrow \varphi(t, x, \lambda) \leq \underline{\lim \varphi\left(t, x_{n}, \lambda_{n}\right) .}
\end{aligned}
$$

On the other hand, again by hypothesis $H(a)$, we have

$$
\varphi\left(t, x, \lambda_{n}\right) \rightarrow \varphi(t, x, \lambda) \text {. }
$$

So we conclude that indeed $\varphi\left(t, \cdot, \lambda_{n}\right) \stackrel{M}{\rightarrow} \varphi(t, \cdot, \lambda)$ in $L^{2}(Z)$. Thus we have satisfied hypothesis $H(\varphi)$.

Next remark that because of hypothesis $H(a)$, for every $\lambda \in B \subseteq E$ compact, we have

$$
\bigcup_{\lambda \in B}\left\{x \in H=L^{2}(Z):\|x\|^{2}+\varphi(t, x, \lambda) \leq \theta\right\}
$$

is bounded in $W^{1,2}(Z)$, hence relatively sequentially weakly compact in there. Since $W^{1,2}(Z)$ embeds compactly in $L^{2}(Z)$ we conclude that hypothesis $H_{c}^{1}(1)$ is satisfied (recall $p \geq 2$ ).

Now let $F: T \times H \times E \rightarrow P_{w k c}(H)$ be defined by

$$
\begin{aligned}
F(t, x, \lambda)=\left\{v \in H=L^{2}(Z):\right. & \xi_{1}(t, z, x(z), \lambda) \leq v(z) \\
& \left.\leq \xi_{2}(t, z, x(z), \lambda) \text { a.e. on } Z\right\} .
\end{aligned}
$$

Then for every $(x, \lambda) \in H \times E$ we have

$$
\begin{aligned}
& \operatorname{Gr} F(\cdot, x, \lambda)=\{(t, v)\in T \times H: v \in F(t, x, \lambda)\} \\
&=\left\{(t, v) \in T \times H: \int_{A} \xi_{1}(t, z, x(z), \lambda) d z \leq \int_{A} v(z) d z\right. \\
&\left.\leq \int_{A} \xi_{2}(t, z, x(z), \lambda) d z \text { for all } A \in B(Z)\right\}
\end{aligned}
$$

(here $B(Z)$ denotes the Borel $\sigma$-field of $Z$ ). Recall that $B(Z)$ is countably generated (separable). So we can find a countable field $\left\{A_{n}\right\}_{n \geq 1} \subseteq B(Z)$ such that $\sigma\left(\left\{A_{n}\right\}_{n \geq 1}\right)=B(Z)$. Then we have

$$
\begin{aligned}
\operatorname{Gr} F(\cdot, x, \lambda)=\bigcap_{n \geq 1}\left\{(t, v) \in T \times H: \int_{A_{n}} \xi_{1}(t, z,\right. & x(z), \lambda) \leq \int_{A_{n}} v(z) d z \\
& \left.\leq \int_{A_{n}} \xi_{2}(t, z, x(z), \lambda) d z\right\} .
\end{aligned}
$$

Note that by Fubini's theorem for every $n \geq 1, t \rightarrow \int_{A_{n}} \xi_{1}(t, z, x(z), \lambda) d z$, $t \rightarrow \int_{A_{n}} \xi_{2}(t, z, x(z), \lambda) d z$ are both measurable. So

$$
\operatorname{Gr} F(\cdot, x, \lambda) \in B(T) \times B(H)
$$


with $B(T)$ (resp. $B(H)$ ) the Borel $\sigma$-field of $T$ (resp. of $H$ ). Then Theorem 4.2 of Wagner [30] tells us that $t \rightarrow F(t, x, \lambda)$ is measurable.

In addition from hypothesis $H(\xi)(2)$ and the definition of the Hausdorff metric, we easily see that for all $\lambda \in B \subseteq E$ compact, we have

$$
h(F(t, x, \lambda), F(t, y, \lambda)) \leq \hat{k}_{B}(t)\|x-y\|_{L^{2}(Z)}
$$

with $\hat{k}_{B}(t)=\left\|k_{B}(t, \cdot)\right\|_{2}$.

Furthermore from hypothesis $H(\xi)(3)$ we have for all $\lambda \in B \subseteq E$ compact $|F(t, x, \lambda)|=\sup \left\{\|v\|_{L^{2}(Z)}: v \in F(t, x, \lambda)\right\} \leq \hat{a}_{B}(t)+\hat{c}_{B}(t)\|x\|_{L^{2}(Z)}$ a.e. on $T$ with $\hat{a}_{B}(t)=\left\|a_{B}(t, \cdot)\right\|_{2}$ and $\hat{c}_{B}(t)=\left\|\hat{c}_{B}(t, \cdot)\right\|_{2}$, while hypothesis $H(\xi)(4)$ gives us the $h$-continuity of $\lambda \rightarrow F(t, x, \lambda)$. So we have satisfied hypothesis $H(F)_{3}$.

Remark that because of hypothesis $H_{0}^{\prime}$, hypothesis $H_{0}$ is automatically satisfied for the initial datum $\lambda \rightarrow \hat{x}_{0}(\lambda)=x_{0}(\cdot, \lambda)$.

Next let $x^{*} \in \partial \varphi(t, x, \lambda) \subseteq L^{2}(Z)$. Immediately we have all $x \in K(t)$. Also if $e \in W^{1, p}(Z)_{+}$(the positive cone in the Sobolev space $\left.W^{1, p}(Z)\right)$, we see that

$$
\lim _{\theta \downarrow 0} \frac{\varphi(t, x+\theta e, \lambda)-\varphi(t, x, \lambda)}{\theta}=\varphi^{\prime}(t, x, \lambda ; e)
$$

with

$$
\varphi^{\prime}(t, x, \lambda ; e)=\int \sum_{z=1}^{N} a(z, \lambda)\left|D_{k} x(z)\right|^{p-2} D_{k} x(z) D_{k} e(z) d z .
$$

From the definition of the subdifferential, we have that

$$
\int_{Z} x^{*}(z) e(z) d z \leq \int_{Z} \sum_{k=1}^{N} a(z, \lambda)\left|D_{k} x(z)\right|^{p-2} D_{k} x(z) D_{k} e(z) d z
$$

for every $e \in W^{1, p}(Z)_{+}$.

In addition, note that if $\theta \in[0,1]$, then $x+\theta(g(t, \cdot)-x) \in K(t)$. So as above, we get that

$$
\begin{aligned}
& \int_{Z} x^{*}(z)(g(t, z)-x(z)) d z \\
& \quad \leq \int_{Z} \sum_{k=1}^{N} a(z, \lambda)\left|D_{k} x(z)\right|^{p-2} D_{k} x(z) D_{k}(g(t, z)-x(z)) d z \\
& \quad \Rightarrow \int_{Z} x^{*}(z)(x(z)-g(t, z)) d z \\
& \quad \geq \int_{Z} \sum_{k=1}^{N} a(z, \lambda)\left|D_{k} x(z)\right|^{p-2} D_{k} x(z) D_{k}(x(z)-g(t, z)) d z .
\end{aligned}
$$

Since $x-g(t, \cdot) \in W^{1, p}(Z)_{+}$, we deduce that

$$
\begin{aligned}
\int_{Z} x^{*}(z)(x(z)-g(t, z)) d z \\
=\int_{Z} \sum_{k=1}^{N} a(z, \lambda)\left|D_{k} x(z)\right|^{p-2} D_{k} x(z) D_{k}(x(z)-g(t, z)) d z .
\end{aligned}
$$


Hence we see that the time-varying subdifferential evolution inclusion on $T \times H$

$$
\left\{\begin{array}{c}
-\dot{x}(t) \in \partial \varphi(t, \\
x(t), \lambda)+F(t, x(t), \lambda) \text { a.e. } \\
x(0)=x_{0}(\lambda)
\end{array}\right\}
$$

is an abstract reformulation of (9). Apply Theorems 3.5 and 3.6 to get the desired Vietoris and $h$-continuity of $S: \Lambda \rightarrow P_{k}\left(C\left(T, L^{2}(Z)\right)\right)$.

Remark. In fact, we can also allow the obstacles to depend on the parameter $\lambda \in E$. So $g: T \times Z \times E \rightarrow \mathbb{R}$ and we assume that when $\lambda_{n} \rightarrow \lambda$ in $E$, then for all $t \in T$ we have $g\left(t, \cdot, \lambda_{n}\right) \stackrel{w}{\rightarrow} g(t, \cdot, \lambda)$ in $W_{0}^{1, s}(Z)$ with $s>p$. If this is the case, then a result of Boccardo-Murat [5] tells us that $K\left(\cdot, \lambda_{n}\right) \stackrel{M}{\rightarrow}$ $K(\cdot, \lambda)$ in $W_{0}^{1, p}(Z)$ and using that, we can establish Theorem 5.1, in this more general context. More general modes of convergence for the obstacles $g(t, \cdot, \lambda)$, involving the notion of $p$-capacity, can be found in Attouch-Picard [3].

(ii) The second example deals with a sequence of optimal control problems with rapidly oscillating coefficients in their dynamics.

So let $T=[0, b]$ and $Z$ be a bounded domain in $\mathbb{R}^{N}$ with a $C^{2}$ boundary $\Gamma=\partial Z$. Let

$$
A_{n}(t) x=-\sum_{i, j=1}^{N} D_{i}\left(a_{i j}^{n}(t, z) D_{j} x(z)\right)
$$

and

$$
A(t) x=-\sum_{i, j=1}^{N} D_{i}\left(a_{i j}(t, z) D_{j} x(z)\right)
$$

where $D_{i}=\frac{\partial}{\partial z_{i}}, D_{j}=\frac{\partial}{\partial z_{j}}$, while $a_{i j}^{n}(t, z)=a_{i j}(t, z)+\frac{1}{2} \cos \left(n z_{2}\right)$ if $i=j=1$, $a_{i j}^{n}(t, z)=a_{i j}(t, z)$ otherwise, for $i, j=1, \ldots, N$. We consider the following sequence of optimal control problems:

$(10)_{n}$

$$
\left\{\begin{array}{c}
\int_{0}^{b} \int_{Z}\left|x(t, z)-v_{n}(t, z)\right|^{2} d z d t+\frac{1}{2} \int_{0}^{b} \int_{Z}|u(t, z)|^{2} d z d t \rightarrow \inf =m_{n} \\
\text { s.t. } \frac{\partial x}{\partial t}+A_{n}(t) x(t, z)=g_{n}(t, z, x(t, z))+B(t, z) u(t, z) \text { a.e. on } T \times Z \\
\left.x\right|_{T \times \Gamma}=0, x(0, z)=x_{0}^{n}(z) \text { a.e. on } Z \\
\|u(t, \cdot)\|_{L^{2}(Z)} \leq r_{n}(t) \text { a.e. with } u(\cdot, \cdot) \text { measurable }
\end{array}\right\}
$$

and the limit problem

(10)

$$
\left\{\begin{array}{c}
\int_{0}^{b} \int_{Z}|x(t, z)-v(t, z)|^{2} d z d t+\frac{1}{2} \int_{0}^{b} \int_{Z}|u(t, z)|^{2} d z d t \rightarrow \inf =m \\
\text { s.t. } \frac{\partial x}{\partial t}+A(t) x(t, z)=g(t, z, x(t, z))+B(t, z) u(t, z) \text { a.e. on } T \times Z \\
\left.x\right|_{T \times \Gamma}=0, x(0, z)=x_{0}(z) \text { a.e. on } Z \\
\|u(t, \cdot)\|_{L^{2}(Z)} \leq r(t) \text { a.e. with } u(\cdot, \cdot) \text { measurable }
\end{array}\right\} .
$$

In this case $E=\mathbb{N}^{*}=\mathbb{N} \cup\{+\infty\}$ (the one-point (Alexandroff) compactification of the locally compact space $\mathbb{N}$ equipped with the discrete metric).

We will need the following hypotheses on the data of $(10)_{n}$ and (10).

$H\left(a_{1}\right): a_{i j} \in L^{\infty}(T \times Z), a_{i j}(t, \cdot) \in C^{0,1}(\bar{Z}), a_{i j}=a_{j i}$, and $c_{1}\|z\|_{N}^{2} \leq$ $\sum_{i, j=1}^{N} a_{i j}(t, z) z_{i} z_{j}$ with $c_{1}>0$ for every $z=\left(z_{i}\right)_{i=1}^{N} \in \mathbb{R}^{N}$ and $\mid a_{i j}(t, z)-$ $a_{i j}(s, z)|\leq k| t-s \mid$ a.e. on $Z$ for all $i, j=1, \ldots, N$. 
$H(g)_{2}: g_{n}, g: T \times Z \times \mathbb{R} \rightarrow \mathbb{R}, n \geq 1$, are functions such that

(1) $(t, z) \rightarrow g_{n}(t, z, x)$ is measurable,

(2) $\left|g_{n}(t, z, x)-g_{n}(t, z, y)\right| \leq k(t, z)|x-y|$ a.e. with $k \in L^{1}(T \times Z)$,

(3) $\left|g_{n}(t, z, x)\right| \leq a(t, z)+c(t, z)|x|$ a.e. with $a, c \in L^{2}(T \times Z)$,

(4) $g_{n}(\cdot, \cdot, x) \rightarrow g(\cdot, \cdot, x)$ a.e. on $T \times Z$ for every $x \in \mathbb{R}$.

$H(r): r_{n} \in L_{+}^{2}, r_{n} \rightarrow r$ in $L^{2}(T, \mathbb{R})$ and $r_{n}(t) \leq a(t)$ a.e. with $a \in L_{+}^{2}$.

$H(v): v_{n} \in L^{2}(T \times Z, \mathbb{R})$ and $v_{n} \rightarrow v$ in $L^{2}(T \times Z, \mathbb{R})$.

$H(B)_{1}: B \in L^{\infty}(T \times Z, \mathbb{R})$.

$H_{0}^{\prime \prime}: x_{0}^{n}, x_{0} \in H_{0}^{1}(Z)$ and $x_{0}^{n} \rightarrow x_{0}$ and $L^{2}(Z)$.

Then we have the following sensitivity result.

Theorem 5.2. If hypotheses $H(a)_{1}, H(g)_{2}, H(r), H(v), H(B)_{1}$, and $H_{0}^{\prime \prime}$ hold, then $m_{n} \rightarrow m$ as $n \rightarrow \infty$.

Proof. Let $H=L^{2}(Z)$ and define $\varphi_{n}, \varphi: T \times H \rightarrow \overline{\mathbb{R}}=\mathbb{R} \cup\{+\infty\}$ as follows:

$$
\begin{aligned}
& \varphi_{n}(t, x)= \begin{cases}\frac{1}{2} \int_{Z} \sum_{i, j=1}^{N} a_{i j}^{n}(t, z) D_{i} x(z) D_{j} x(z) d z & \text { if } x \in H_{0}^{1}(Z), \\
+\infty & \text { otherwise, }\end{cases} \\
& \varphi(t, x)= \begin{cases}\frac{1}{2} \int_{Z} \sum_{i, j=1}^{N} a_{i j}(t, z) D_{i} x(z) D_{j} x(z) d z & \text { if } x \in H_{0}^{1}(Z), \\
+\infty & \text { otherwise. }\end{cases}
\end{aligned}
$$

Then we have $\partial \varphi_{n}(t, x)=A_{n}(t) x$ and $\partial \varphi(t, x)=A(t) x$ with domain $D=H_{0}^{1}(Z) \cap H^{2}(Z)$ (regularity theory of elliptic equations, since $\Gamma=\partial Z$ is by hypothesis $\left.C^{2}\right)$. It is easy to check that $\varphi_{n}(t, \cdot), \varphi(t, \cdot) \in \Gamma_{0}(H)$. Also using hypothesis $H(a)$ and Poincare's inequality, we get that $\left|\varphi_{n}(t, x)-\varphi_{n}(s, x)\right| \leq$ $k|t-s|\|x\|_{H_{0}^{1}(Z)}^{2} \leq \hat{k}|t-s| \varphi(t, x)$ for some $\hat{k}>0$. So hypotheses $H(\varphi)(1)$ and (2) have been satisfied. Next observe that $\left\{\frac{1}{2} \cos \left(n z_{2}\right)\right\}_{n \geq 1}$ is a sequence of $C^{\infty}$ functions, which converges to 0 in $H^{-1}(Z)$ but not in $L^{2}(Z)$ (indeed from the Riemann-Lebesgue lemma we know that $\frac{1}{2} \cos \left(n z_{2}\right) \stackrel{w}{\rightarrow} 0$ in $L^{2}(Z)$ and recall that $L^{2}(Z)$ embeds compactly in $H^{-1}(Z)=H_{0}^{1}(Z)^{*}$, to get $\frac{1}{2} \cos \left(n z_{2}\right) \rightarrow 0$ as $\left.H^{-1}(Z)\right)$. Then for all $t \in T, A_{n}(t) \rightarrow A(t)$ in $H^{-1}(Z)$ and so from Theorem 13.12, p. 159 of Dal Maso [9] (see also Attouch [2], Proposition 3.69, p. 379), we have that for all $t \in T, \varphi_{n}(t, \cdot) \stackrel{M}{\rightarrow} \varphi(t, \cdot)$ in $L^{2}(Z)$. So we have satisfied hypothesis $H(\varphi)$. Furthermore, since $H_{0}^{1}(Z)$ embeds compactly into $L^{2}(Z)$, we see that hypothesis $H_{c}(1)$ holds.

Next let $\hat{g}_{n}, \hat{g}: T \times L^{2}(Z) \rightarrow L^{2}(Z)$ be defined by

$$
\hat{g}_{n}(t, x)(z)=g_{n}(t, z, x(z)) \text { and } \hat{g}(t, x)(z)=g(t, z, x(z))
$$

(the Nemitsky (superposition) operators corresponding to the functions $g_{n}(t, z, x)$ and $\left.g(t, z, x)\right)$. Then using hypothesis $H(g)_{2}$, we see that $\hat{g}_{n}(t, x)$ $\rightarrow \hat{g}(t, x)$ in $L^{2}(Z)=H$. So we have satisfied hypothesis $H(g)$.

Also let $U_{n}(t)=\left\{u \in L^{2}(Z)=Y:\|u\|_{2} \leq r_{n}(t)\right\}$ and $U(t)=\left\{u \in L^{2}(Z)\right.$ : $\left.\|u\|_{2} \leq r(t)\right\}$. Then because of hypothesis $H(r)$, we see that hypothesis $H(U)$ is satisfied. 
Next let $L_{n}, L: T \times H \times Y \rightarrow \mathbb{R}$ (recall that $H=L^{2}(Z)=Y$ ) be defined by

$$
L_{n}(t, x, u)=\int_{Z}\left|x(z)-v_{n}(t, z)\right|^{2} d z+\frac{1}{2} \int_{Z}|u(z)|^{2} d z
$$

and

$$
L(t, x, u)=\int_{Z}|x(z)-v(t, z)|^{2} d z+\frac{1}{2} \int_{Z}|u(z)|^{2} d z .
$$

Using hypothesis $H(v)$, we can easily check that hypothesis $H(L)$ is valid.

Finally, let $\hat{B}(t)(\cdot) \in \mathscr{L}(H)$ be defined by $\widehat{B}(t) u(\cdot)=B(t, \cdot) u(\cdot)$ and note that because of hypothesis $H_{0}^{\prime \prime}$, hypothesis $H_{0}$ holds.

Now rewrite $(10)_{n}$ and (10) in the following equivalent forms with dynamics described by subdifferential evolution equations (here $\hat{x}_{0}^{n}=x_{0}^{n}(\cdot)$ and $\hat{x}_{0}=$ $\left.x_{0}(\cdot)\right)$ :

$$
\left\{\begin{array}{c}
J_{n}(x, u)=\int_{0}^{b} L_{n}(t, x(t), u(t)) d t \rightarrow \inf =m_{n} \\
\text { s.t. }-\dot{x}(t) \in \partial \varphi_{n}(t, x(t))+\hat{g}_{n}(t, x(t))+\widehat{B}(t) u(t) \text { a.e. } \\
x(0)=\hat{x}_{0}^{n} \\
u(t) \in U_{n}(t) \text { a.e., } u(\cdot) \text { measurable }
\end{array}\right\} .
$$

and the limit problem

$$
\left\{\begin{array}{c}
J(x, u)=\int_{0}^{b} L(t, x(t), u(t)) d t \rightarrow \inf =m \\
\text { s.t. }-\dot{x}(t) \in \partial \varphi(t, x(t))+\hat{g}(t, x(t))+\widehat{B}(t) u(t) \text { a.e. } \\
x(0)=\hat{x}_{0} \\
u(t) \in U(t) \text { a.e. } u(\cdot) \text { measurable }
\end{array}\right\}
$$

Invoking Theorem 4.1, we finally get that $m_{n} \rightarrow m$.

Remark. In addition, we know that if $\left\{x_{n}\right\}_{n \geq 1} \subseteq C\left(T, L^{2}(Z)\right)$ is a sequence of optimal states for problem $(10)_{n}$, then we can find a subsequence $\left\{x_{n_{k}}\right\}_{k \geq 1}$ such that $x_{n_{k}} \rightarrow x$ in $C\left(T, L^{2}(Z)\right)$ and $x$ is optimal for the limit problem (10). This follows from the proof of Theorem 3.5, from which we get that $\left\{x_{n}\right\}_{n \geq 1}$ is relatively compact in $C\left(T, L^{2}(Z)\right)$, and from Theorem 4.2, which assures that the limit state $x(\cdot, \cdot)$ is optimal for (10).

(iii) Suppose $N=1$ and let $Z=(0,1)$. Now the dynamical equations in problems $(10)_{n}$ and $(10)$ have the forms

$$
\frac{\partial x}{\partial t}-\frac{\partial}{\partial z}\left(a_{n}(t, z) \frac{\partial x}{\partial z}\right)=g_{n}(t, z, x(t, z))+B(t, z) u(t, z) \text { a.e. on } T \times Z
$$

and

$$
\frac{\partial x}{\partial t}-\frac{\partial}{\partial z}\left(a(t, z) \frac{\partial x}{\partial z}\right)=g(t, z, x(t, z))+B(t, z) u(t, z) \text { a.e. on } T \times Z
$$

with $a_{n}, a \in L^{\infty}(T \times Z), a_{n}(t, \cdot), a(t, \cdot) \in \operatorname{Lip}[0,1], 0<\hat{c} \leq a_{n}(t, z)$, $a(t, z)$ and $\left|a_{n}(t, z)-a_{n}(s, z)\right|,|a(t, z)-a(s, z)| \leq \hat{k}|t-s|$ a.e. on $Z$ with $\hat{k}>0$. Assume that for all $t \in T, \frac{1}{a_{n}(t, \cdot)} \stackrel{w^{*}}{\rightarrow} \frac{1}{a(t, \cdot)}$ in $L^{\infty}(Z)$. Then from Dal Maso [9] (see Example 6.6, p. 55, and Theorem 13.12, p. 159) we have that $\varphi_{n}(t, \cdot) \stackrel{M}{\rightarrow} \varphi(t, \cdot)$ in $L^{2}(Z)$, where as before $\varphi_{n}, \varphi: T \times L^{2}(Z) \rightarrow \overline{\mathbb{R}}=$ $\mathbb{R} \cup\{+\infty\}$ are defined by

$$
\varphi_{n}(t, x)= \begin{cases}\int_{0}^{1} a_{n}(t, z)\left(\frac{d x(z)}{d z}\right)^{2} d z & \text { if } x \in H_{0}^{1}(Z), \\ +\infty & \text { otherwise }\end{cases}
$$


and

$$
\varphi(t, x)= \begin{cases}\int_{0}^{1} a(t, z)\left(\frac{d x(z)}{d z}\right)^{2} d z & \text { if } x \in H_{0}^{1}(Z) \\ +\infty & \text { otherwise }\end{cases}
$$

Hence in this case, if the other hypotheses remain the same (with $Z=(0,1)$ ) we have that $m_{n} \rightarrow m$. For example we can have $a_{n}(z)=1+e^{-n z}$ and $a(z)=1$.

Remark. The above convergence hypothesis on the coefficients $a_{n}(t, z)$ and $a(t, z)$ guarantees that the corresponding partial differential operators converge in the $G$-sense to the limit one (see Spagnolo [28] and Zhikov-Kozlov-Oleinik [33]). In general the one-dimensional case is better than the multidimensional one allowing for more general convergence modes on the coefficients because of the following elementary fact: if $\left\{u_{n}\right\}_{n \geq 1} \subseteq L^{p}(0,1), n \geq 1$, with $1<p<\infty$ and $u_{n} \stackrel{w}{\rightarrow} u$ in $L^{p}(0,1)$, while $u_{n}^{\prime} \rightarrow u^{\prime}$ in $W^{-1, p}(0,1)$, then $u_{n} \stackrel{s}{\rightarrow} u$ in $L^{p}(0,1)$. To see this assume without any loss of generality that $u=u^{\prime}=0$. From the representation theorem for Sobolev spaces (see for example Adams [1], Theorem 3.8, p. 48), we know that we can find $u_{n} \in L^{p}(0,1)$ such that $v_{n}^{\prime}=v_{n}^{\prime}$ and $\left\|u_{n}^{\prime}\right\|_{W^{-1, p}(0,1)}=\left\|v_{n}\right\|_{p}$. Since $Z=(0,1)$, we have $u_{n}=v_{n}+c_{n}$, $c_{n}=$ constant . So $v_{n} \rightarrow 0$ in $L^{p}(0,1)$ and because $u_{n} \stackrel{w}{\rightarrow} 0$ in $L^{p}(0,1)$, we get that $c_{n} \rightarrow 0$ in $\mathbb{R}$. So finally $u_{n} \rightarrow 0$ in $L^{p}(0,1)$.

(iv) Our formulation also incorporates "differential variational inequalities", namely evolution inclusions of the form

$$
\left\{\begin{array}{c}
-\dot{x}(t) \in N_{K(t, \lambda)}(x(t))+F(t, x(t), \lambda) \text { a.e. } \\
x(0)=x_{0}(\lambda) .
\end{array}\right\}
$$

Here $N_{K(t, \lambda)}(x)$ denotes the normal cone to the closed, convex set $K(t, \lambda)$ at $x$. Recall that $N_{K(t, \lambda)}(x)=T_{K(t, \lambda)}(x)^{-}$, where $T_{K(t, \lambda)}(x)$ is the tangent cone to $K(t, \lambda)$ at $x$ and $T_{K(t, \lambda)}(x)^{-}$is its negative polar (see for example Laurent [20]).

These inclusions arise in theoretical mechanics in the study of elastoplastic systems (see Moreau [22]). Furthermore, if $K(t, \lambda)=K(\lambda)$ (i.e. the set $K$ is independent of time) and $H=\mathbb{R}^{N}$, then Cornet [8] proved that inclusion (11) above is in fact equivalent to the following projected differential inclusion:

$$
\left\{\begin{array}{c}
-\dot{x}(t) \in \operatorname{proj}\left(-F(t, x(t), \lambda) ; T_{K(\lambda)}(x)\right) \text { a.e. } \\
x(0)=x_{0}(\lambda)
\end{array}\right\} .
$$

Here $\operatorname{Proj}\left(\cdot ; T_{K(\lambda)}(x)\right)$ denotes the metric projection on the closed, convex cone $T_{K(\lambda)}(x)$. Cornet [8] and Henry [14] indicated that differential inclusions like (12) above arise naturally in mathematical economics in the study of planning procedures. More generally, if a dynamic system has state constraints, in describing the effect of the constraints on the dynamic equation, it can be assumed in many cases that the velocity $\dot{x}(t)$ is projected at each time instant on the set of allowed directions towards the constraint set at the point $x(t)$. This then leads us to the multivalued Cauchy problem (12), which as we already mentioned, is equivalent to (11). This is true for electrical networks with diode nonlinearities (for details and additional examples we refer to the book of Krasnosel'skii-Pokrovskii [18]). 
Recall (see for example Laurent [20]), that if $\varphi(t, x, \lambda)=\delta_{K(t, \lambda)}(x)=0$ if $x \in K(t, \lambda)$ and $+\infty$ otherwise (the indicator function of the closed and convex set $K(t, \lambda) \subseteq H)$, then $\partial \varphi(t, x, \lambda)=N_{K(t, \lambda)}(x)$. So problem (11) fits in the general framework of this paper.

To illustrate the use of problems like (11) (and also (12)), consider the following differential variational inequality on $T \times \mathbb{R}^{N}$ :

(13)

$\left\{\begin{array}{c}x(\cdot) \in C\left(T, \mathbb{R}^{N}\right), \theta_{1}(\lambda) \leq x(t) \leq \theta_{2}(\lambda) \text { for all } t \in T, x(0)=x_{0}(\lambda) \\ \dot{x}(t) \in F(t, x(t), \lambda) \text { a.e. on } T_{1}(\lambda)=\left\{s \in T=[0, b]: \theta_{1}(\lambda)<x(s)<\theta_{2}(\lambda)\right\} \\ -\dot{x}(t) \in F(t, x(t), \lambda)-\mathbb{R}_{+}^{N} \text { a.e. on } T_{2}(\lambda)=\left\{s \in T=[0, b]: \theta_{1}(\lambda)=x(s)\right\} \\ -\dot{x}(t) \in F(t, x(t), \lambda)+\mathbb{R}_{+}^{N} \text { a.e. on } T_{3}(\lambda)=\left\{s \in T=[0, b]: \theta_{2}(\lambda)=x(s)\right\}\end{array}\right\}$.

We will need the following hypotheses on the data of (13):

$H(\theta): \theta_{1}, \theta_{2}: E \rightarrow \mathbb{R}^{N}$ are continuous functions such that for all $\lambda \in E, \theta_{1}(\lambda)$ $\leq \theta_{2}(\lambda)$.

$H(F)_{4}: F: T \times \mathbb{R}^{N} \times E \rightarrow P_{k c}\left(\mathbb{R}^{N}\right)$ is a multifunction such that

(1) $t \rightarrow F(t, x, \lambda)$ is measurable,

(2) $h(F(t, x, \lambda), F(t, y, \lambda)) \leq k_{B}(t)\|x-y\|$ a.e. for all $\lambda \in B \subseteq E$ compact and with $k_{B}(\cdot) \in L_{+}^{1}$,

(3) $|F(t, x, \lambda)|=\sup \{\|v\|: v \in F(t, x, \lambda)\} \leq a_{B}(t)+c_{B}(t)\|x\|$ a.e. for all $\lambda \in B \subseteq E$ compact and with $a_{B}, c_{B} \in L_{+}^{2}$,

(4) $\lambda \rightarrow F(t, x, \lambda)$ is $h$-continuous.

$H_{0}^{\prime \prime \prime}: \lambda \rightarrow x_{0}(\lambda)$ is continuous from $E$ into $\mathbb{R}^{N}$ and for all $\lambda \in E, \theta_{1}(\lambda) \leq$ $x_{0}(\lambda) \leq \theta_{2}(\lambda)$.

Let $K(\lambda)=\left\{v \in \mathbb{R}^{N}: \theta_{1}(\lambda) \leq v \leq \theta_{2}(\lambda)\right\}$. So

$$
K(\lambda)=\left[\theta_{1}(\lambda)+\mathbb{R}_{+}^{N}\right] \cap\left[\theta_{2}(\lambda)-\mathbb{R}_{+}^{N}\right]
$$

where $\mathbb{R}_{+}^{N}=\left\{v \in \mathbb{R}^{N}: v=\left(v_{i}\right)_{i=1}^{N}\right.$ and $v_{i} \geq 0$ for all $\left.i=1, \ldots, N\right\}$ (the positive orthant of $\mathbb{R}^{N}$ ).

Then combining hypothesis $H(\theta)$ with Proposition 2.3 of DeBlasi-Pianigiani [10], we have that $\lambda \rightarrow K(\lambda)$ is continuous from $E$ into $P_{k c}\left(\mathbb{R}^{N}\right)$. If $x \in$ int $K(\lambda)$, then $T_{K(\lambda)}(x)=\mathbb{R}^{N}$ and so $N_{K(\lambda)}(x)=\{0\} ;$ if $x=\theta_{1}(\lambda)$, then $T_{K(\lambda)}(x)=\mathbb{R}_{+}^{N}$ and so $N_{K(\lambda)}(x)=-\mathbb{R}_{+}^{N}$; and finally if $x=\theta_{2}(\lambda)$, then $T_{K(\lambda)}(x)=-\mathbb{R}_{+}^{N}$ and so $N_{K(\lambda)}(x)=\mathbb{R}_{+}^{N}$. Thus if we set $\varphi(x, \lambda)=\delta_{K(\lambda)}(x)$, then $\partial \varphi(x, \lambda)=N_{K(\lambda)}(x)$ and so problem (13) is equivalent to the following differential variational inequality:

$$
\left\{\begin{array}{c}
-\dot{x}(t) \in N_{K(\lambda)}(x(t))+F(t, x(t), \lambda) \text { a.e. } \\
x(0)=x_{0}(\lambda)
\end{array}\right\} .
$$

Denote the solution set of (13) by $S(\lambda) \subseteq C\left(T, \mathbb{R}^{N}\right)$. Note that the finite dimensionality of the state space guarantees that hypothesis $H_{c}$ is satisfied (in fact both $H_{c}(1)$ and $H_{c}(2)$ hold; cf. hypothesis $H(\theta)$ ). So we can apply Theorems 3.5 and 3.6 and get:

Theorem 5.3. If hypotheses $H(\theta), H(F)_{4}$, and $H_{0}^{\prime \prime \prime}$ hold, then

$$
S: E \rightarrow P_{k}\left(C\left(T, \mathbb{R}^{N}\right)\right)
$$

is both Vietoris and h-continuous. 
For the more general time-dependent system (11) defined on an infinite dimensional separable Hilbert space $H$, we need the following hypothesis on the set $K(t, \lambda)$ :

$H(K): K: T \times E \rightarrow P_{f c}(H)$ is a multifunction such that $h\left(K\left(t^{\prime}, \lambda\right), K(t, \lambda)\right) \leq$ $\int_{t}^{t^{\prime}} v(s) d s$ for all $0 \leq t \leq t^{\prime} \leq b$ and all $\lambda \in E$ and with $v(\cdot) \in L^{2}(T)$, and furthermore if $\lambda_{n} \rightarrow \lambda$ in $E$, then $K\left(t, \lambda_{n}\right) \stackrel{M}{\rightarrow} K(t, \lambda)$ (here $\stackrel{M}{\rightarrow}$ denotes the Mosco convergence; i.e. $w-\varlimsup$ $\underline{\lim } K(t, \lambda)$, see Section 2).

So if we set $\varphi(t, x, \lambda)=\delta_{K(t, \lambda)}(x)$, then we see that hypotheses $H(\varphi)(1)$ and (2) are satisfied with $g_{r}(t)=\int_{0}^{t} v(s) d s, t \in T, \dot{g}_{r}(\cdot)=v(\cdot) \in L^{2}(T)$, $\beta=2, \alpha=0$ and $K_{r}=1$ for all $r>0$. Also from Mosco [23], we know that since $K\left(t, \lambda_{n}\right) \stackrel{M}{\rightarrow} K(t, \lambda)$ for $\lambda_{n} \rightarrow \lambda$ in $E$, then $\varphi\left(t, \cdot, \lambda_{n}\right) \stackrel{M}{\rightarrow} \varphi(t, \cdot, \lambda)$. Thus from Theorems 3.5 and 3.6 we have:

Theorem 5.4. If hypotheses $H(K), H(F)_{3}, H_{c}^{1}$, and $H_{0}$ hold, then $S: E \rightarrow$ $P_{k}(C(T, H))$ is both Vietoris and h-continuous.

(v) As a final application we consider a class of multivalued parabolic partial differential equations, which are of interest in the study of free boundary problems. So let $T=[0, b]$ and $Z$ be a bounded domain in $\mathbb{R}^{N}$, with a $C^{2}$ boundary $\Gamma=\partial Z$. Also let $\gamma$ be a maximal monotone subset of $\mathbb{R} \times \mathbb{R}$. Hence $\gamma$ is automatically cyclically maximal monotone (see Brezis [6], p. 43) and so there exists $j \in \Gamma_{0}(\mathbb{R})$ (unique up to an additive constant) such that $\gamma=\partial j$. We assume that $0 \in D(\gamma)$ and $j \geq 0$. We consider the following problem:

$\left\{\begin{array}{c}\frac{\partial x}{\partial t}-\operatorname{div}(a(t, z, \lambda) D x)+\gamma(x(t, z)) \ni f(t, z) \text { a.e. on } T \times Z \\ \left.x\right|_{T \times \Gamma}=0, x(0, z)=x_{0}(z, \lambda) \text { a.e. on } Z, \\ f(t, \cdot) \in\left\{h \in L^{2}(Z): h(z)=\int_{Z} k\left(t, z, z^{\prime}\right) g\left(z^{\prime}, x\left(z^{\prime}\right), \lambda\right) d z^{\prime} \text { a.e. on } Z\right\}\end{array}\right\}$.

We will need the following hypotheses on the data:

$H(a)_{2}: a: T \times Z \times E \rightarrow \mathbb{R}, a(\cdot, \cdot, \lambda)$ is measurable, $0<m_{1 B}<a(t, z, \lambda) \leq$ $m_{2 B}$ for all $\lambda \in B \subseteq E$ compact $|a(t, z, \lambda)-a(s, z, \lambda)| \leq \hat{k}|t-s|$ a.e. on $Z$ and if $\lambda_{n} \rightarrow \lambda$ in $E$, then $a\left(\cdot, \cdot, \lambda_{n}\right) \rightarrow a(\cdot, \cdot, \lambda)$ a.e. on $T \times Z$.

$H(k): k \in L^{2}(T \times Z \times Z)$.

$H(g)_{3}: g: T \times Z \times \mathbb{R} \times E \rightarrow \mathbb{R}$ is a function such that

(1) $(t, z) \rightarrow g(t, z, x, \lambda)$ is measurable,

(2) $|g(t, z, x, \lambda)-g(t, z, y, \lambda)| \leq k_{B}(t, z)|x-y|$ a.e. for all $\lambda \in$ $B \subseteq E$ compact and with $k_{B} \in L^{2}(T \times Z)$,

(3) $|g(t, z, x, \lambda)| \leq a_{B}(t, z)+c_{B}(t, z)|x|$ a.e. for all $\lambda \in B \subseteq E$ compact and with $a_{B}, c_{B} \in L^{2}(T \times Z)$,

(4) $\lambda \rightarrow g(t, z, x, \lambda)$ is continuous.

$H_{0}^{(\text {iv) }}: \hat{x}_{0}(\lambda)=x_{0}(\cdot, \lambda) \in H_{0}^{1}(Z), j\left(x_{0}(\cdot, \lambda)\right) \in L^{1}(Z)$ and for every $B \subseteq E$ compact we have $\sup _{\lambda \in B} \int_{Z} j\left(x_{0}(z, \lambda)\right) d z<\infty$.

We denote the solution set of (14) by $S(\lambda) \subseteq C\left(T, L^{2}(Z)\right)$. We have the following result concerning this solution multifunction : 
Theorem 5.5. If hypotheses $H(a)_{2}, H(k), H(g)_{3}$, and $H_{0}^{(\mathrm{iv})}$ hold, then $S: E \rightarrow$ $P_{k}\left(C\left(T, L^{2}(Z)\right)\right)$ is both Vietoris and h-continuous.

Proof. Let $H=L^{2}(Z)$ and define $\varphi: T \times H \times E \rightarrow H$ by

$$
\varphi(t, x, \lambda)=\left\{\begin{array}{c}
\frac{1}{2} \int_{Z} a(t, z, \lambda)\|D x(z)\|_{N}^{2} d z+\int_{Z} j(x(z)) d z \\
\quad \text { if } x \in H_{0}^{1}(Z), j(x(\cdot)) \in L^{1}(Z) \\
+\infty \text { otherwise. }
\end{array}\right\}
$$

From Brezis [7], Example 6, we know that $\varphi(t, \cdot, \lambda) \in \Gamma_{0}(H)$ and

$$
\partial \varphi(t, x, \lambda)=\left\{u \in L^{2}(Z): u(z) \in \gamma(x(z))-\operatorname{div}(a(t, z, \lambda) x(z)) \text { a.e. on } Z\right\} \text {. }
$$

Then as before we can easily check that $\varphi(t, x, \lambda)$ satisfies $H(\varphi)(1)$ and (2). Furthermore, hypothesis $H(a)_{2}$ combined with Theorem 5.14, p. 51, and Theorem 13.12, p. 159, of Dal Maso [9] tells us that if $\lambda_{n} \rightarrow \lambda$ in $E$, then $\varphi\left(t, \cdot, \lambda_{n}\right) \stackrel{M}{\rightarrow} \varphi(t, \cdot, \lambda)$ in $L^{2}(Z)$. So hypothesis $H(\varphi)$ is valid.

Also let $F: T \times H \times E \rightarrow P_{w k c}(H)$ be defined by

$$
F(t, x, \lambda)=\left\{h \in H: h(z)=\int_{Z} k\left(t, z, z^{\prime}\right) g\left(z^{\prime}, x\left(z^{\prime}\right), \lambda\right) d z^{\prime} \text { a.e. on } Z\right\} \text {. }
$$

Using hypotheses $H(k)$ and $H(g)_{3}$, it is easy to check that $H(F)_{3}$ is satisfied. Furthermore, note that for every $t \in T$ and every $\lambda \in E$, the operator $R_{t, \lambda}: H \rightarrow H$ defined by $R_{t, \lambda}(x)(z)=\int_{Z} k\left(t, z, z^{\prime}\right) g\left(z^{\prime}, x\left(z^{\prime}\right), \lambda\right) d z^{\prime}$ is the composition of a Nemitsky (superposition) operator which is continuous and bounded and a Hilbert-Schmidt integral operator which is compact. So $R_{t, \lambda}(\cdot)$ is compact and in addition because of hypothesis $H(g)_{3}(3)$, we see that hypothesis $H_{c}(2)$ holds. Finally hypothesis $H_{0}^{(\mathrm{iv})}$ implies hypothesis $H_{0}$.

Rewrite problem (14) in the following equivalent form:

$$
\left\{\begin{array}{c}
-\dot{x}(t) \in \partial \varphi(t, x(t), \lambda)+F(t, x(t), \lambda) \text { a.e. } \\
x(0)=\hat{x}_{0}(\lambda)
\end{array}\right\} .
$$

Apply Theorems 3.5 and 3.6 to get the desired continuities of $\lambda \rightarrow S(\lambda)$.

\section{REFERENCES}

1. R. A. Adams, Sobolev spaces, Academic Press, New York, 1975.

2. H. Attouch, Variational convergence for functions and operators, Pitman, London, 1984.

3. $\mathrm{H}$. Attouch and C. Picard, Inequations variationelles avec obstacles et espaces fonctionelles en theorie du potentiel, Applicable Anal. 12 (1981), 287-306.

4. E. Balder, Necessary and sufficient conditions for $L^{1}$-strong-weak lower semicontinuity of integral functionals, Nonlinear Anal. 11 (1987), 1399-1404.

5. L. Boccardo and F. Murat, Nouveaux résultas de convergence dans de problèmes unilatéraux, Nonlinear Partial Differential Equations and their Applications, College de France, Vol. II (H. Brezis and J.-L. Lions, eds), Research Notes in Math., Pitman, London, 1982, pp. 64-85.

6. H. Brezis, Operateurs maximaux monotones et semigroupes de contractions dans les espaces de Hilbert, North-Holland, Amsterdam, 1973.

7. differential equations, Contributions to Nonlinear Functional Analysis (E. Zarantonello, ed.), Academic Press, 1971, pp. 101-156. 
8. B. Cornet, Existence of slow solutions for a class of differential inclusions, J. Math. Anal. Appl. 96 (1983), 130-147.

9. G. Dal Maso, An introduction to Г-convergence, Birkhäuser, Boston, 1993.

10. F. S. DeBlasi and G. Pianigiani, Remarks on Hausdorff continuous multifunctions and selections, Comment. Math. Univ. Carolin. 24 (1983), 553-561.

11. F. S. DeBlasi and J. Myjak, On continuous approximations for multifunctions, Pacific J. Math. 123 (1986), 9-31.

12. N. Dunford and J. Schwartz, Linear operators I, Wiley, New York, 1958.

13. J. Dugundji, Topology, Allyn and Bacon, Boston, 1966.

14. C. Henry, Differential equations with discontinuous right-hand side for planning procedures, J. Econom. Theory 4 (1972), 545-551.

15. N. Kenmochi, Some nonlinear parabolic variational inequalities, Israel J. Math. 22 (1975), 305-331.

16. plications, Bull. Fac. Ed. Chiba Univ. 30 (1981), 1-87.

17. E. Klein and A. Thompson, Theory of correspondences, Wiley, New York, 1988.

18. M. A. Krasnosel'skii and A. V. Pokrovskii, Systems with hysteresis, Springer-Verlag, Berlin, 1988.

19. D. Kravvaritis and N. S. Papageorgiou, Multivalued perturbations of subdifferential type evolution equations in Hilbert space, J. Differential Equations 76 (1988), 238-255.

20. P.-J. Laurent, Approximation et optimisation, Hermann, Paris, 1972.

21. T.-C. Lim, On fixed point stability for set-valued contractive mappings, with applications to generalized differential equations, J. Math. Anal. Appl. 110 (1985), 436-441.

22. J.-J. Moreau, Evolution problem associated with a moving convex set in a Hilbert space, J. Differential Equations 26 (1977), 374-374.

23. U. Mosco, Convergence of convex sets and solutions of variational inequalities, Adv. Math. 3 (1969), 510-585.

24. N. S. Papageorgiou, Convergence theorems for Banach space-valued integrable multifunctions, Internat. J. Math. and Math. Sci. 10 (1987), 433-442.

25. On measurable multifunctions with applications to random multivalued equations, Math. Japon. 32 (1987), 437-464.

26. $\ldots$ On the solution set of evolution inclusions driven by time dependent subdifferentials, Math. Japon. 37 (1992), 1087-1099.

27. _ Nonconvex evolution inclusions generated by subdifferential operators, Publ. Math. Debrecen (to appear).

28. S. Spagnolo, Convergence of parabolic equations, Boll. Un. Mat. Ital. 14 (1977), 547-568.

29. A. Vasilev, Continuous dependence of the solutions of differential inclusions on the parameter, Ukranian Math. J. 35 (1983), 520-524.

30. D. Wagner, Survey of measurable selection theorems, SIAM J. Control Optim. 15 (1977), 859-903.

31. Y. Yamada, On evolution equations generated by subdifferential operators, J. Fac. Sci. Univ. Tokyo Sect. IA 23 (1976), 491-515.

32. S. Yotsutani, Evolution equations associated with the subdifferentials, J. Math. Soc. Japan 31 (1978), 623-646.

33. V. Zhikov, S. Kozlov, and O. Oleinik, G-convergence of parabolic operators, Russian Math. Surveys 36 (1981), 9-60.

Department of Mathematics, National Technical University, Zografou Campus, ATHENS 15773, GReECE

Department of Applied Mathematics, Florida Institute of Technology, 150 West UnIVERSity BlVD., MelbourNe, Florida 32901-6988 